\title{
Cellular Mechanism of Mouse Atrial Development
}

\section{Yunping Li}

Key Laboratory of Regenerative Medicine of Ministry of Education, College of Life Science and Technology, Jinan University, Guangzhou, China

Email: liyunping1993@126.com

How to cite this paper: Li, Y.P. (2022) Cellular Mechanism of Mouse Atrial Development. Open Journal of Regenerative Medicine, 11, 1-24.

https://doi.org/10.4236/ojrm.2022.111001

Received: December 10, 2021

Accepted: January 16, 2022

Published: January 19, 2022

Copyright (C) 2022 by author(s) and Scientific Research Publishing Inc. This work is licensed under the Creative Commons Attribution International License (CC BY 4.0).

http://creativecommons.org/licenses/by/4.0/

\begin{abstract}
During the development of mammalian heart, the left and right atria play an important role in cardiovascular circulation. The embryonic atrium is mainly formed by the differentiation of progenitor cells and the proliferation of cardiomyocytes, while the postnatal atrium is primarily shaped by the increase in the volume of cardiomyocytes. Cell proliferation and differentiation of atrial development is the basis for its functions such as "blood reservoir" and "supplementary pump". Deep understanding the cellular mechanism of atrial development is imperative to explore the causes of common congenital arrhythmia heart diseases such as atrial fibrillation. We used genetically engineered mouse reproduction knowledge, lineage tracing method based on CreloxP system, molecular biology and immunofluorescence technology to track the cardiomyocyte lineage of Nppa-GFP mouse line with stereo fluorescence microscope and ultra-high-speed confocal microscope. Besides the atrium of Nppa-CreER; Rosa26 tdTomato mouse was examined during embryonic (E10.5 - E18.5) and postnatal (P0, P3, P5, P7, P14, P28, P8w) stage. Immunofluorescence results revealed that Nppa-positive cells labeled TNNI3-positive cardiomyocytes and protruded into the atrial cavity at the beginning of E11.5 E12.0 and during subsequent development to form Nppa-positive myocardial trabeculae. Thick comb-shaped myocardium was observed after birth, and we suspect that this was particularly important for the normal contractile activity and pumping function of the atrium. Additionally, non-single origin of Nppapositive trabecular myocardiocytes were revealed through Tamoxifen-induced lineage tracing experiment. Our findings reveal proliferation dynamics and non-comprehensive fate decisions of cardiomyocytes that produce the distinct architecture of the atrium chamber.
\end{abstract}

\section{Keywords}

Atrium, Cellular Mechanism, Lineage Tracing, Immunofluorescence, Mouse 


\section{Introduction}

Cells continuously differentiate, multiply, and specialize into cells and structures during the early development of the heart, with different functions and finally form a four-chambered heart with atria and ventricles [1]. The abnormal formation of structures or structure remodeling in this process due to various reasons may lead to congenital heart disease such as atrial fibrillation. Therefore, it is necessary to reveal the normal process of the heart developing into four ordinary chambers, which includes the cell fate and molecular mechanism of the chambers. This article focuses on the fate of atrial cardiomyocytes.

As the first organ to form and function during embryonic development, the heart has gone through the heart tube from the beginning, the stage of looping to the final stage of septum formation. The whole process is also affected by molecular mechanism regulation and intro-environmental factors. In the past few years, scientists have used various lineage tracing strategies to study the fate of precursor cells or progenitor cells in the early stages of heart development [2] [3] [4].

On the eighth day of embryonic heart development, the atria are formed by the heart mesoderm at the posterior site and the ventricles are formed by the cranial heart mesoderm. There have been numerous reports on some fundamental differences between atria and ventricles [1] [5] [6]. In particular, humans know more about the morphogenesis of the ventricle [7] [8], but how the atrial chamber is built has not been explored at a comparable level. Here, we used a NppaCreER mouse line for inducible lineage tracing. Our instant lineage tracing approach identifies Nppa-expressing cardiomyocytes that are labeled during tamoxifen treatment. We first revealed the specific pattern of Nppa-positive cardiomyocytes during morphogenesis of atrial myocardium, which may give impetus to understand the cellular mechanism of atrial development to explore the causes of common congenital arrhythmia heart diseases.

\section{Results}

\section{Morphology of myocardium revealed by Nppa spatial-temporal expres-} sion

We used the Nppa-GFP knock-in mouse line where Nppa was a specific molecular marker for atrial myocardium and green fluorescent protein (GFP) worked as a surrogate for endogenous Nppa expression [8] [9].

E10.5. The stereo fluorescence image showed that green fluorescent protein (GFP) was expressed on the outer wall of the atrium edge, and the fluorescence intensity was weak. The immunofluorescence image revealed that the left and right atriums of the embryonic heart were formed during this period, and the thinnest part of the atrium wall was only one cell thick. The thicker part contained 2 - 3 cells. The cardiomyocytes have not yet invaded the heart cavity, the atrial wall inner surface was relatively flat, and no myocardial trabeculae were detected in the cavity part (Figure 1). 

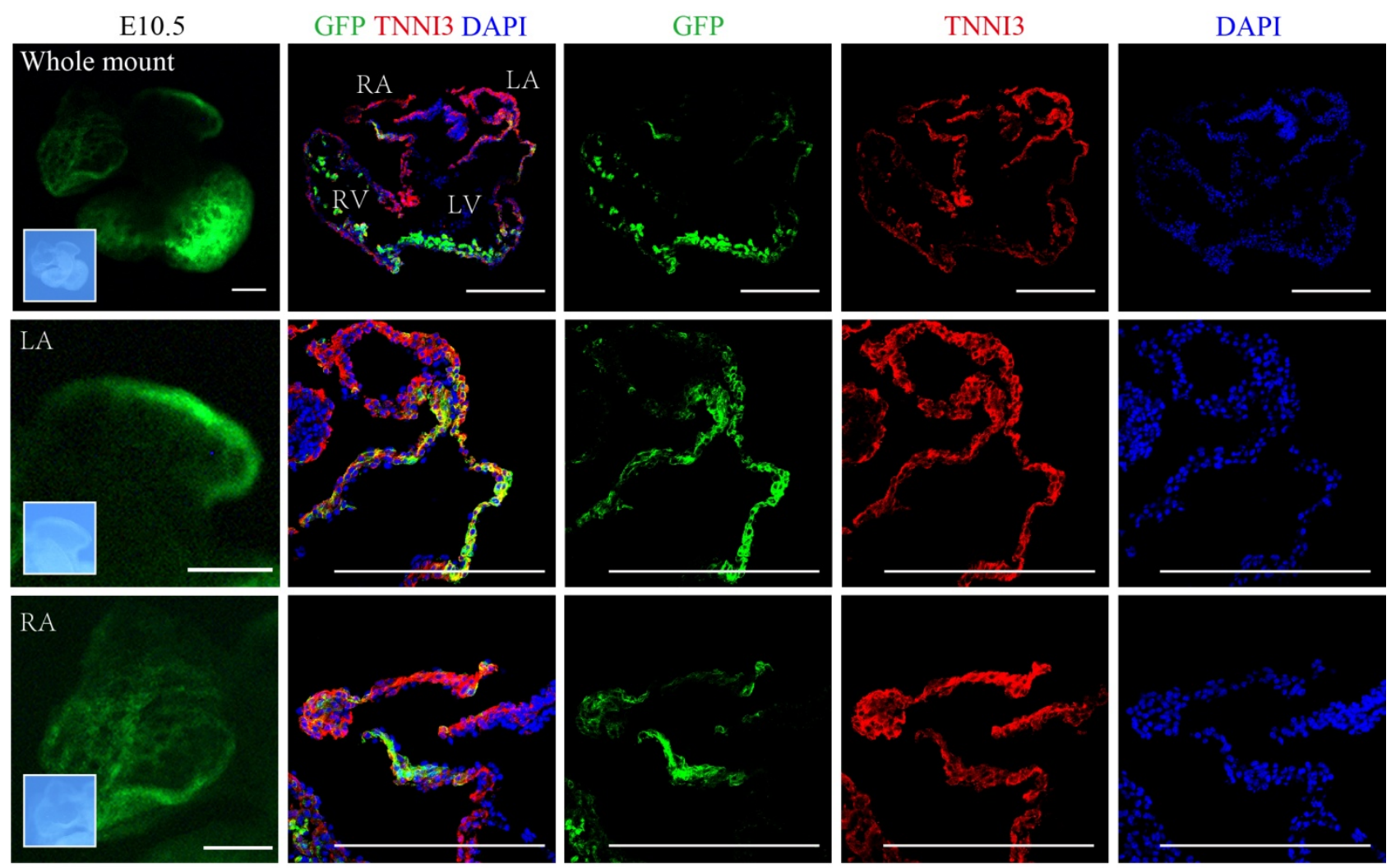

Scale bar: $250 \mu \mathrm{m}$

Figure 1. Co-staining of GFP (Alexa Fluor 488) and TNNI3 (Alexa Fluor 555) on E10.5 (Embryonic day 10.5) Nppa-GFP mouse heart section. Inserts are bright-field images of same hearts or portions. RA, right atria. DAPI, nuclear staining. LA, left atria. RV, right ventricle. LV, left ventricle. Scale bars are indicated. For brevity and clarity, the following images Figures 2-17 will no longer be annotated as they are similar in structure except the timepoint, and the abbreviations below have the same meaning.

E11.5. GFP immunoreactive cells (hereinafter referred to as GFP-positive cells) were distributed on the left and right atrium walls. The atrial wall inner surface was basically smooth. While at the uppermost of the right atrium cardiomyocytes were protruding into the cavity. An extremely small amount of myocardium existed in trabeculae. Compared to the previous period the wall of the left and right atrium was thicker. 3 - 4 layers of cardiomyocytes constituted of atrium wall and the left atrium wall was thicker than the right atrium wall with $3-6$ layers of cardiomyocytes (Figure 2).

E12.0. The GFP-positive cells in the atrium wall invaded into the atrial cavity at the top of the right atrium and increase in number (compared to E11.5), while the atrial wall cavity surface of the left atrium was still relatively smooth without the appearance of myocardial trabeculae (Figure 3).

E12.5. The number of GFP-positive myocardial trabecular cells in the right atrium continued to increase. The GFP-positive cells on the top of the left atrium protruded into the atrial cavity and began to form a small amount of trabecular myocardium (Figure 4).

E13.5. The number of GFP-positive trabecular cardiomyocytes in the right atrial cavity has increased significantly and was connected to form a network 

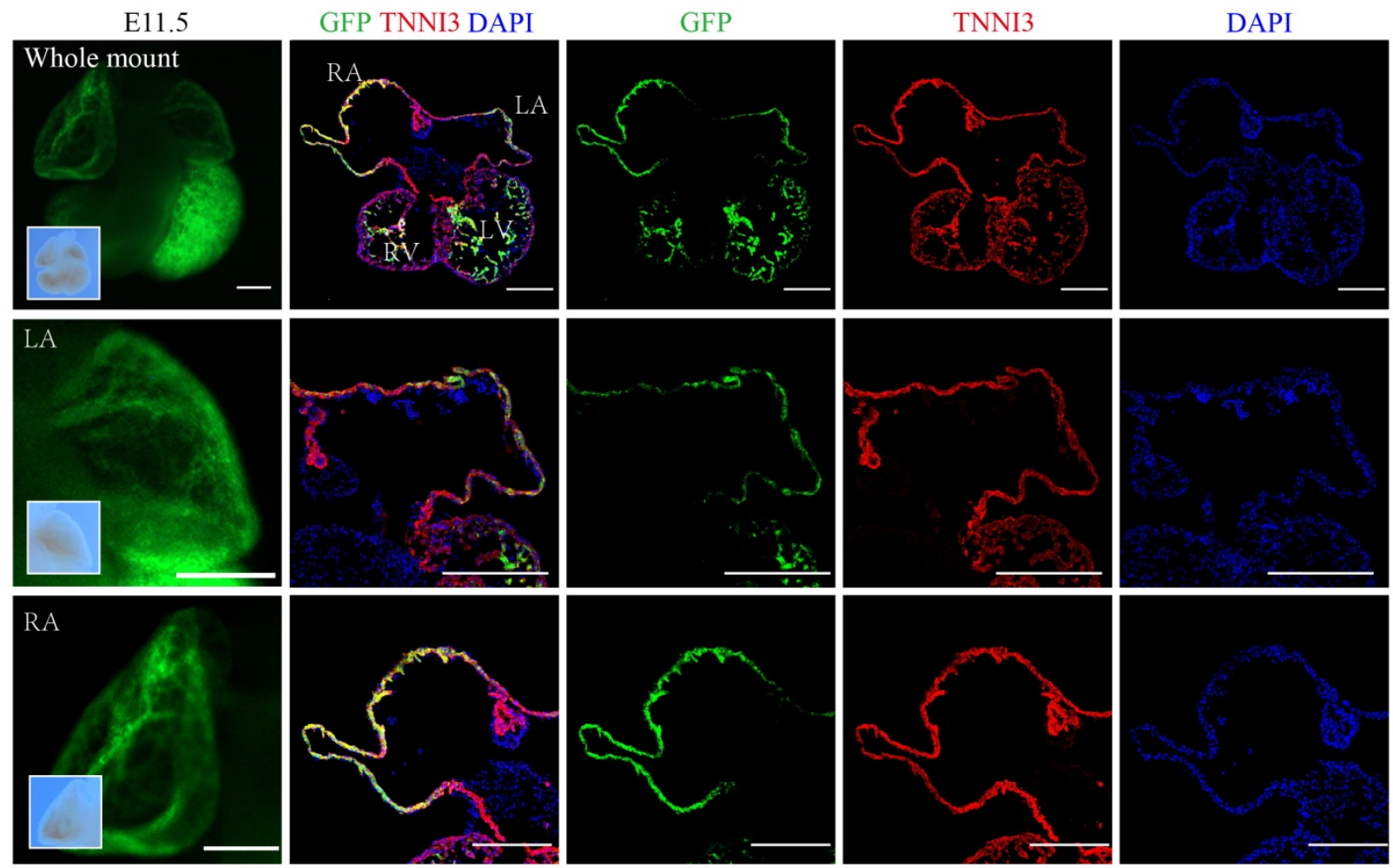

Scale bar: $250 \mu \mathrm{m}$

Figure 2. Immunostaining image of E11.5 Nppa-GFP heart section.

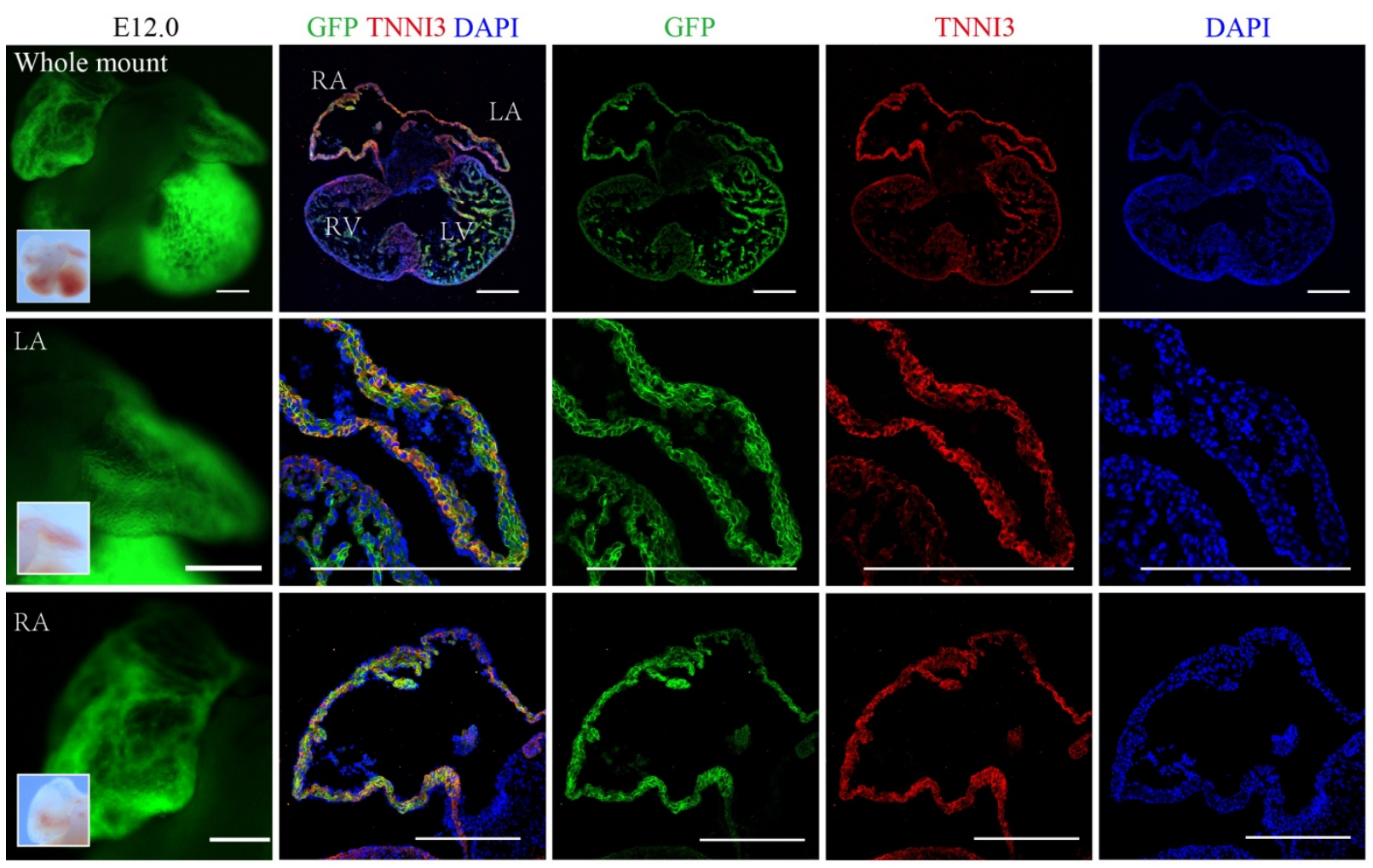

Scale bar: $250 \mu \mathrm{m}$

Figure 3. Immunostaining image of E12.0 Nppa-GFP heart section. 

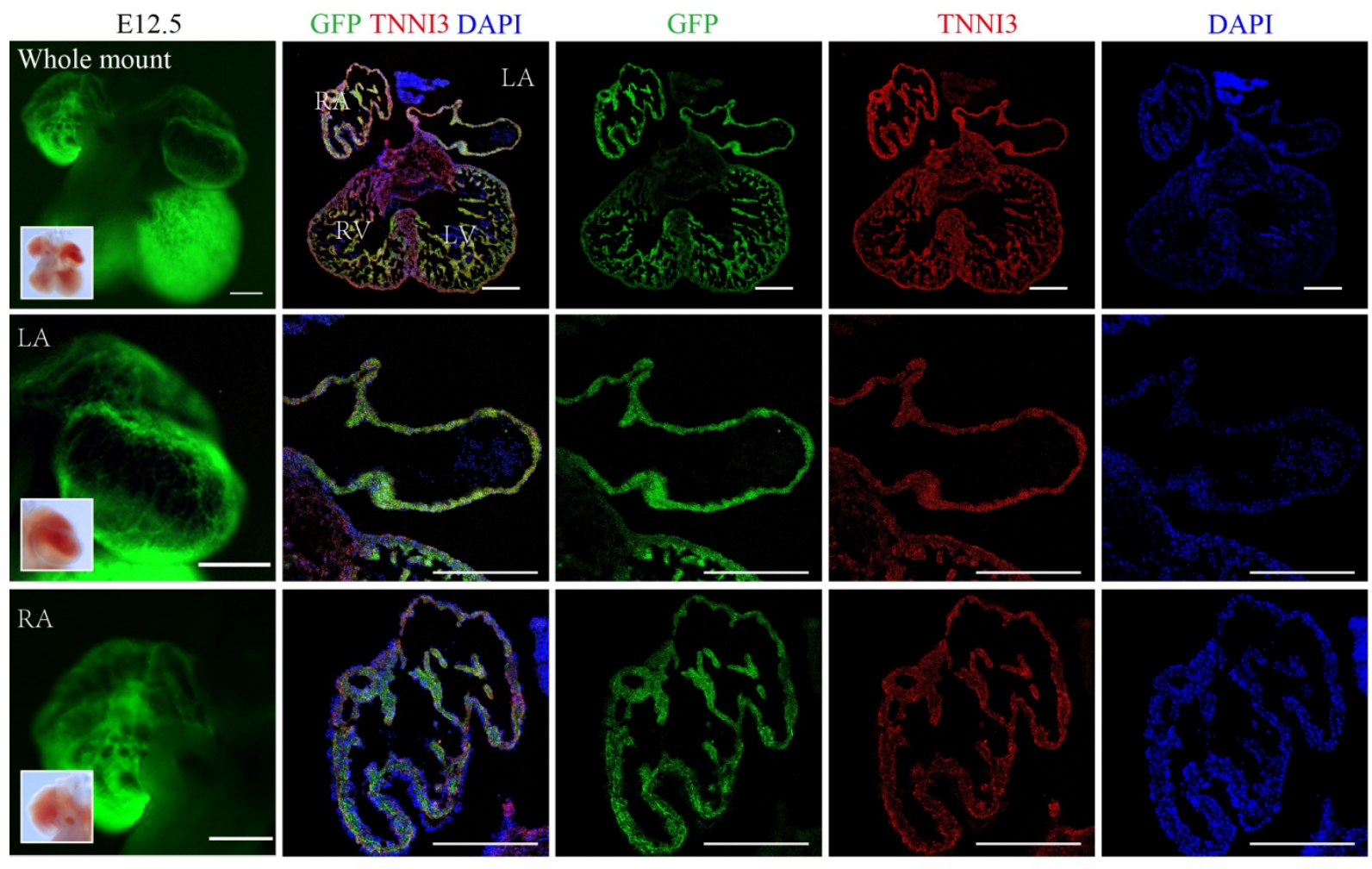

Scale bar: $250 \mu \mathrm{m}$

Figure 4. Immunostaining image of E12.5 Nppa-GFP heart section.

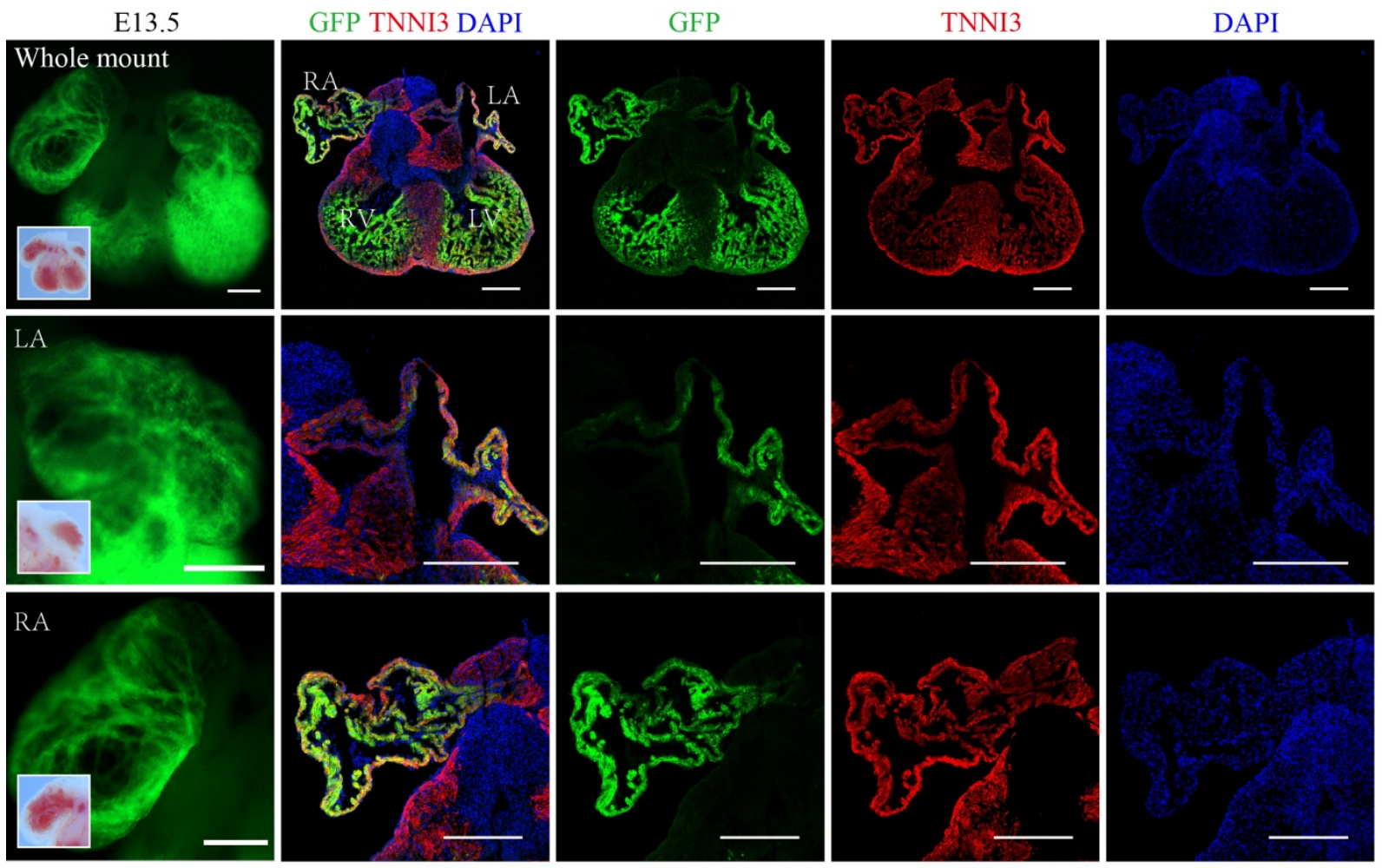

Scale bar: $250 \mu \mathrm{m}$

Figure 5. Immunostaining image of E13.5 Nppa-GFP heart section. 
structure. Although the left atrium has not yet formed a trabecular network structure, there were still a small number of trabecular cardiomyocytes from the top and lateral sides of the atrium (Figure 5).

E14.5. GFP principally became visible in the right atrial reticular trabecular myocardium. While GFP was relatively rare in the atrial wall, and the GFPpositive cells in the atrial wall were close to the side of the atrial cavity or close to the endocardium lateral distribution. The number of left atrial trabecular myocardial cells was increasing (Figure 6).

E15.5. The fluorescence intensity of GFP expressed in the left and right atria attained the highest level during this period. GFP-positive cardiomyocytes form a directional comb-shaped trabecular meshwork in the right atrium and the posterior cavity. The comb-shaped myocardium separated the right atrium into the front and rear cavities. There were fewer tangled myocardial trabeculae in the lateral of the atrium. In the left atrium, the myocardial trabeculae are mainly distributed in the lateral. At the same time, there was a small amount of trabecular myocardium protruding from the atrium wall into the cavity at the top (Figure 7).

E16.5. As the development progresses, the cardiomyocytes in the top, anterior, central, and posterior chambers of the right atrium are connected to form a continuous network of myocardial tissue. The number of trabecular myocardial cells in the left atrium continued to increase and formed a small area of continuous

E14.5
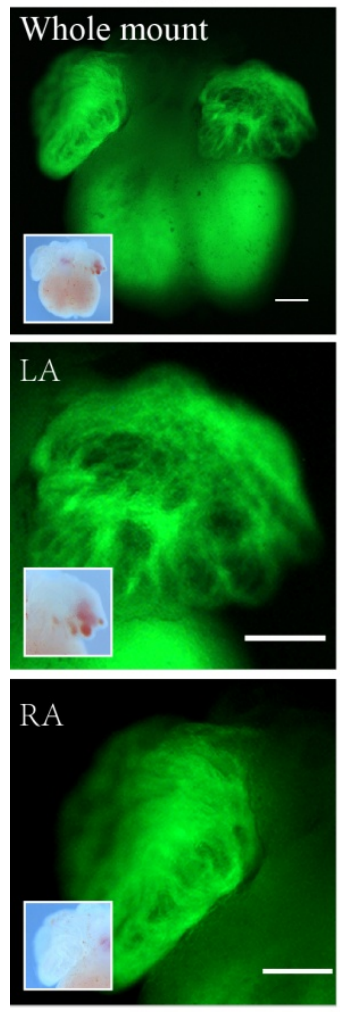

GFP TNNI3 DAPI
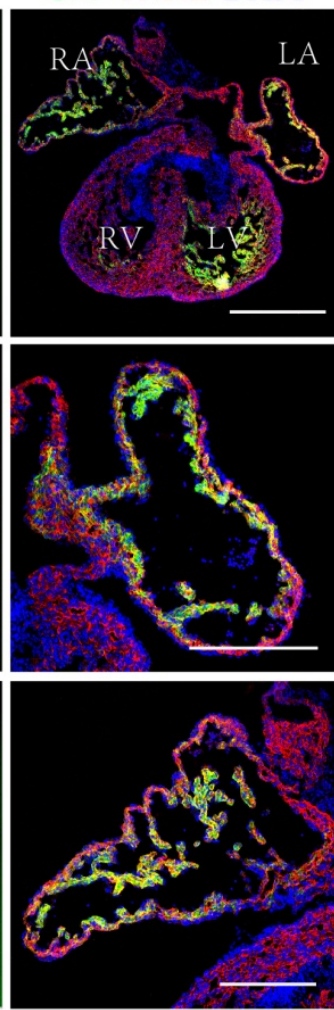

GFP
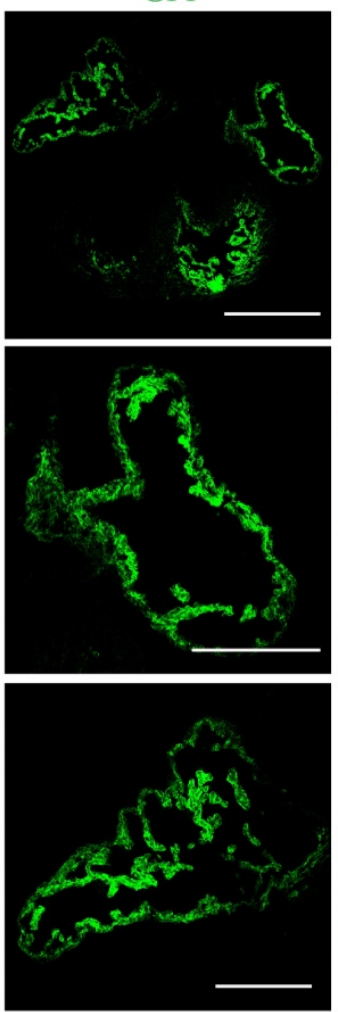

TNNI3
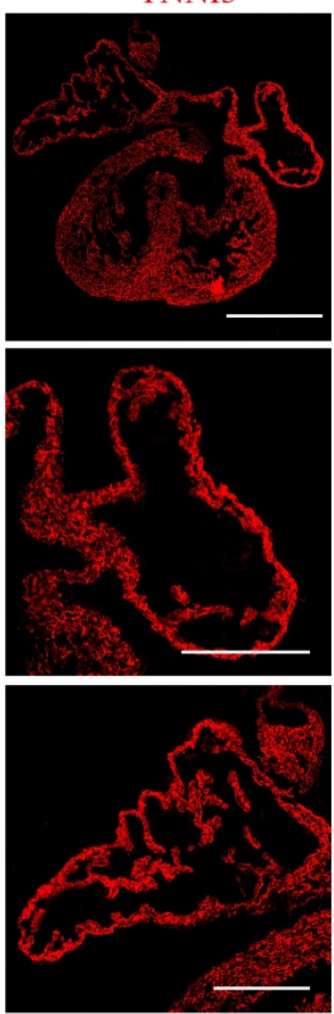

DAPI
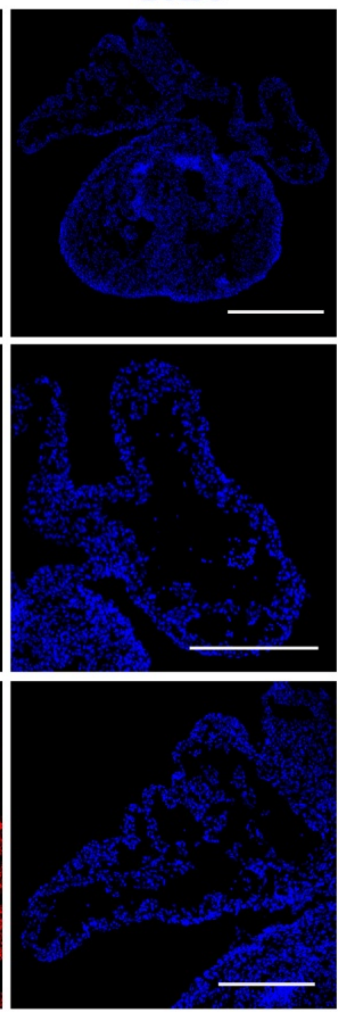

Scale bar: $250 \mu \mathrm{m}$

Figure 6. Immunostaining image of E14.5 Nppa-GFP heart section. 

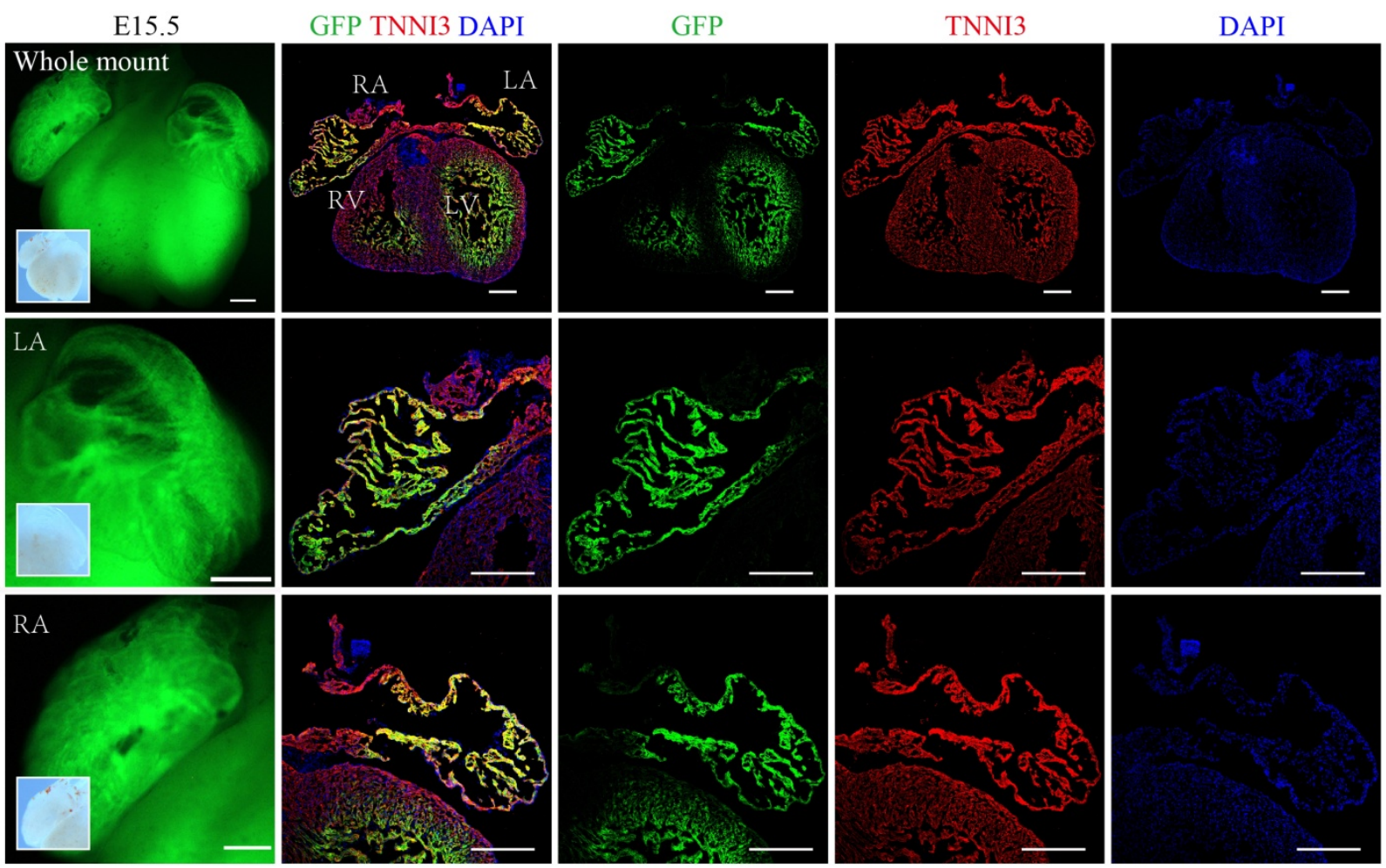

Scale bar: $250 \mu \mathrm{m}$

Figure 7. Immunostaining image of E15.5 Nppa-GFP heart section.

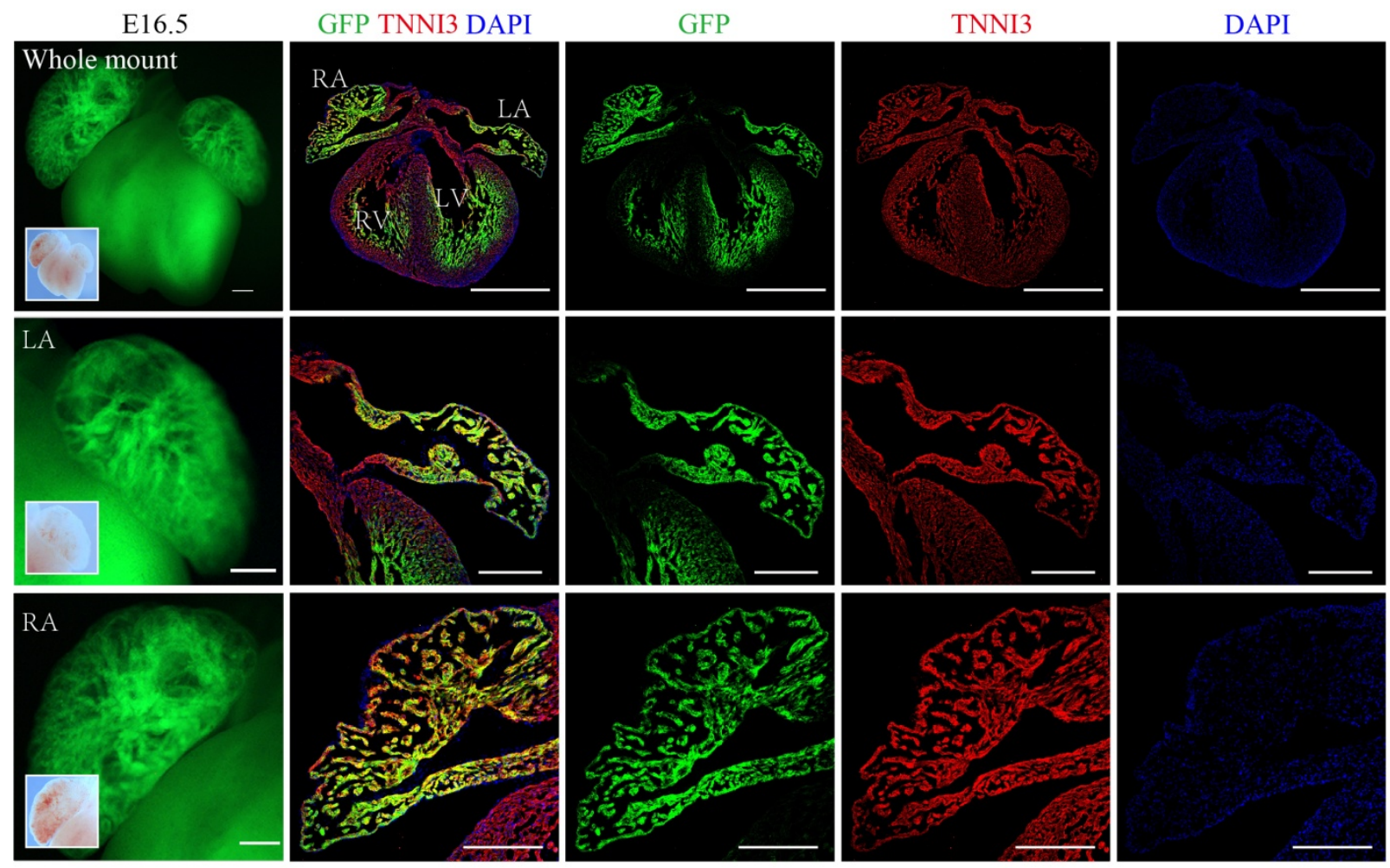

Scale bar: $250 \mu \mathrm{m}$

Figure 8. Immunostaining image of E16.5 Nppa-GFP heart section. 

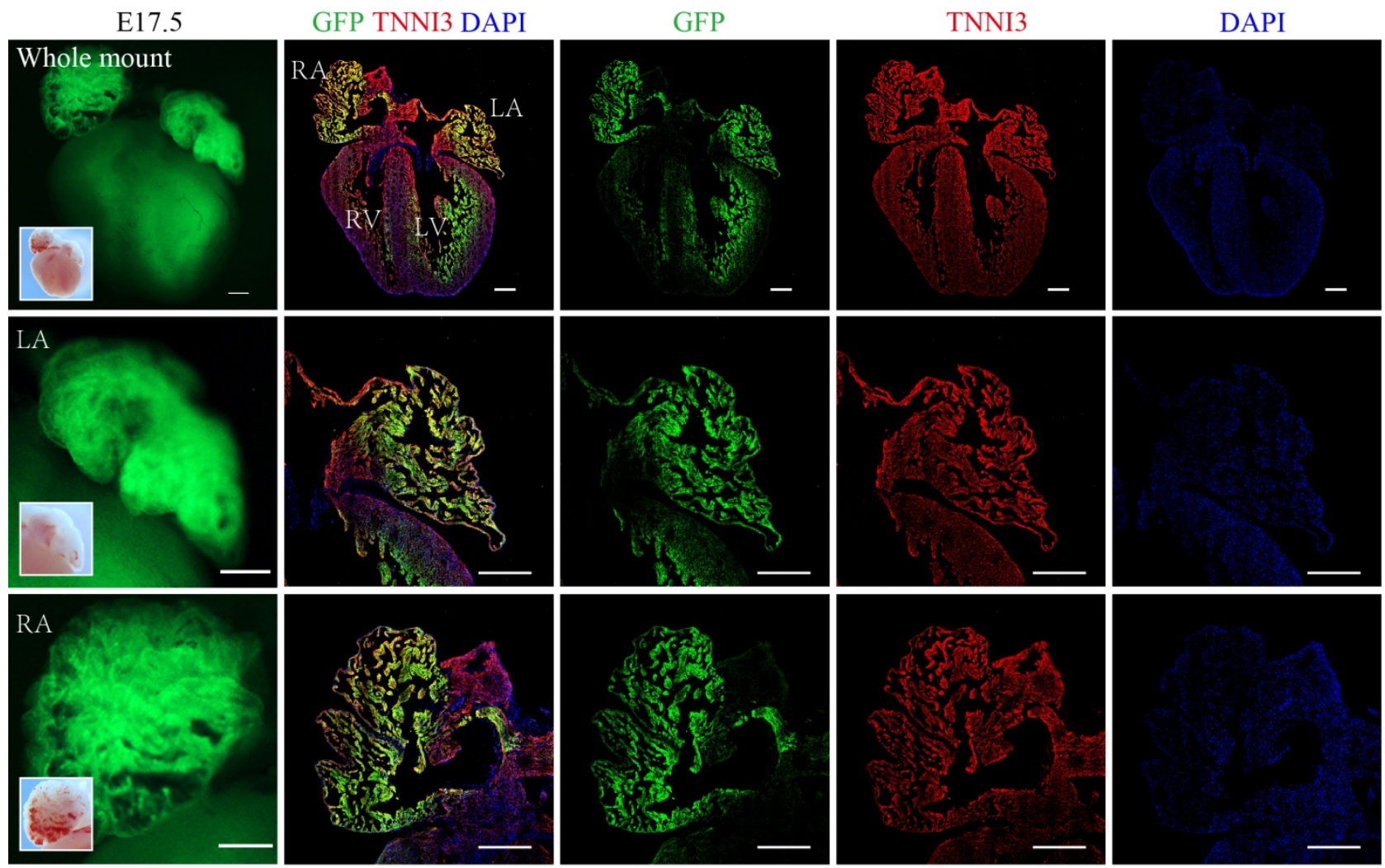

Scale bar: $250 \mu \mathrm{m}$

Figure 9. Immunostaining image of E17.5 Nppa-GFP heart section.

trabecular muscles. While only a tiny trabecular myocardium in the lateral part was detected, which seemed to form a pocket-sized cavity here (Figure 8).

E17.5. GFP-positive cells almost filled the entire right atrium cavity, leaving only a small part of the cavity close to the midline of the atrial septum. The left atrium had a similar situation as there was still a narrow cavity structure in the lateral part (Figure 9).

E18.5. Compared with the narrow and pointed anterior and triangular whole before, the shape of the right atrium was chock-full and three-dimensionally trapezoidal, and a continuous layer of GFP-positive myocardial trabeculae was formed by the atrial wall at the bottom of the atrium. Thicker myocardial tissue was visible as the number of left atrial trabecular myocardial cells increased. But the proportion of trabecular muscles did not bourgeon due to the boost in the volume of the entire atrium (Figure 10).

P0. The left atrium was elongated and narrower than the right atrium. GFPpositive myocardial cells encircled the left and right atria in a loop or ring shape (Figure 11).

P3. The trabecular muscles were interwoven into a mesh-like myocardium in the left and right atriums and continuously thickened. The right atrium was divided into two comparable chambers located in the central and rear part and the front part respectively (Figure 12). 


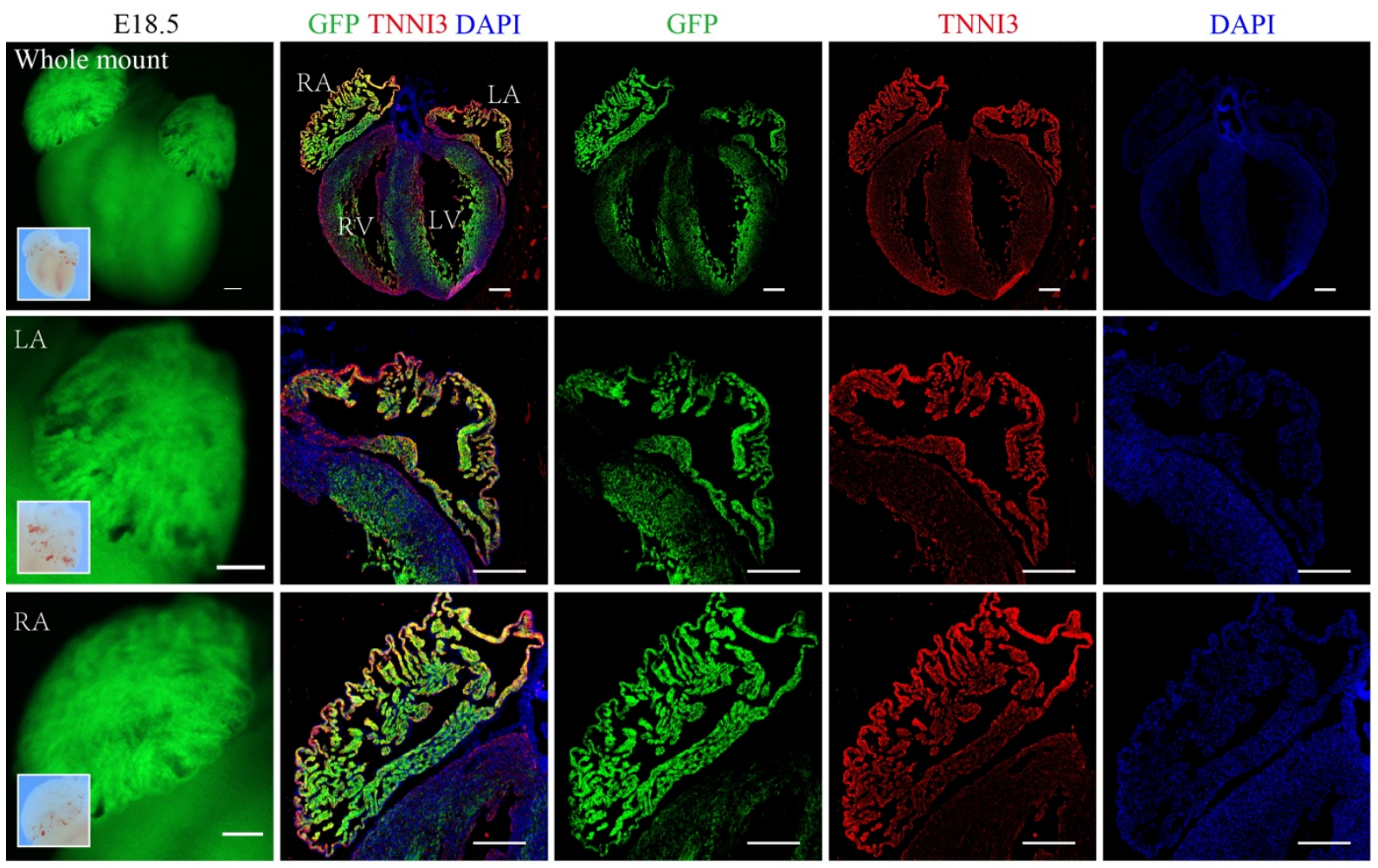

Scale bar: $250 \mu \mathrm{m}$

Figure 10. Immunostaining image of E18.5 Nppa-GFP heart section.

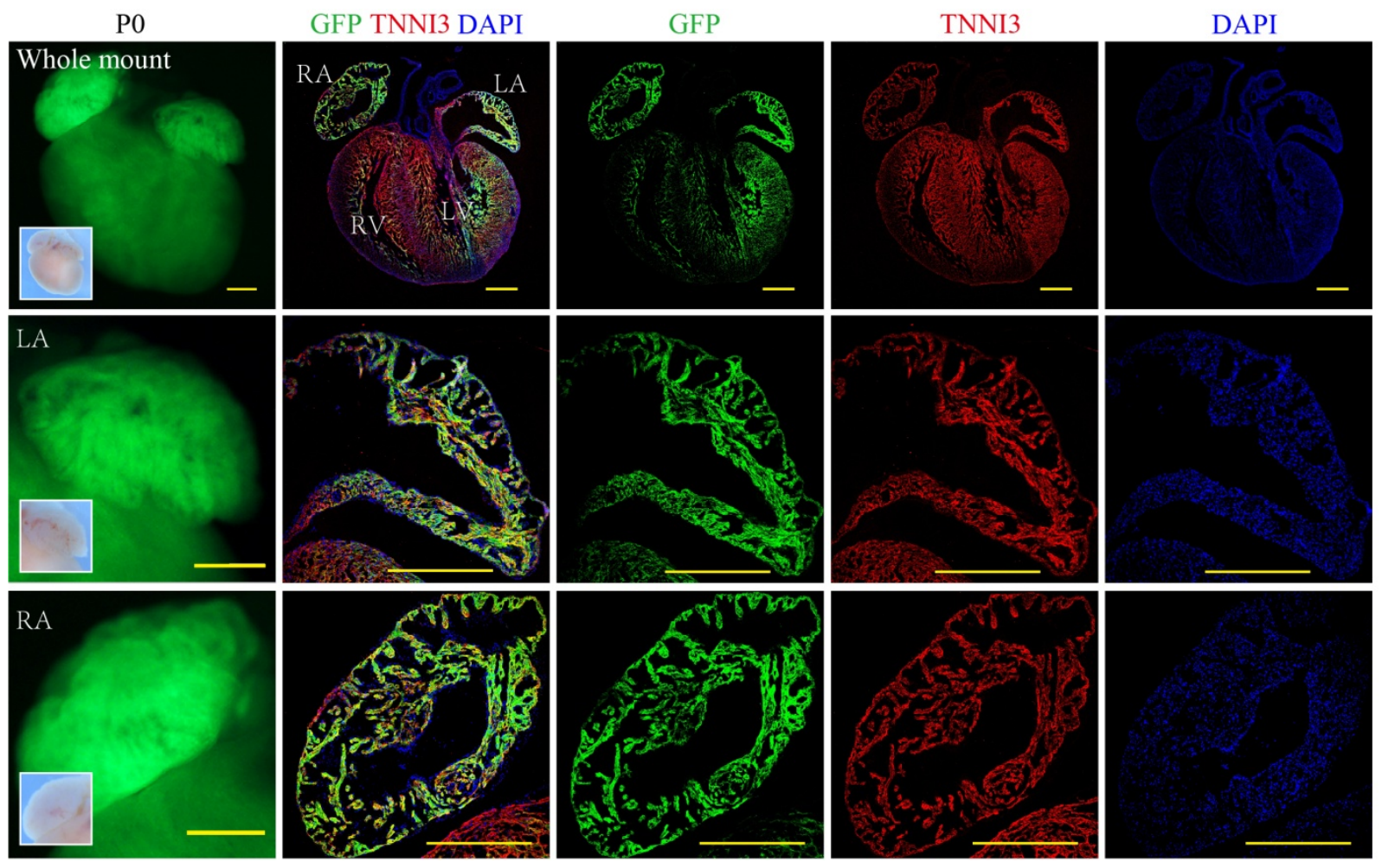

yellow scale bar: $500 \mu \mathrm{m}$

Figure 11. Immunostaining image of P0 Nppa-GFP heart section. 


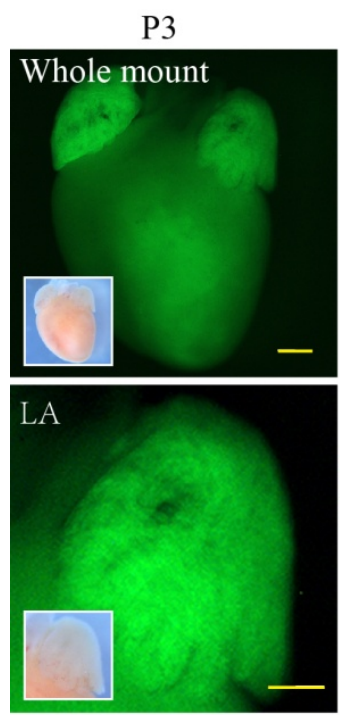

GFP TNNI3 DAPI
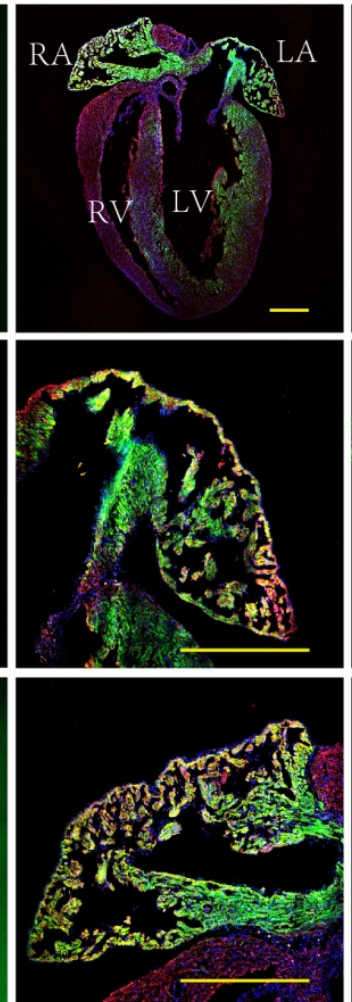

GFP
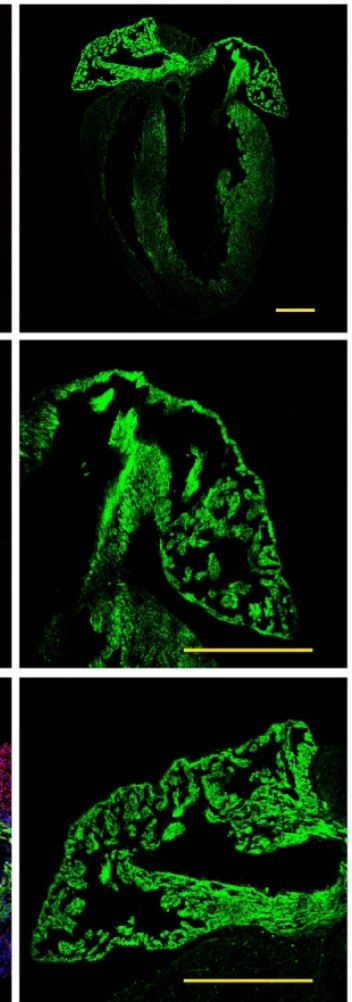

TNNI3
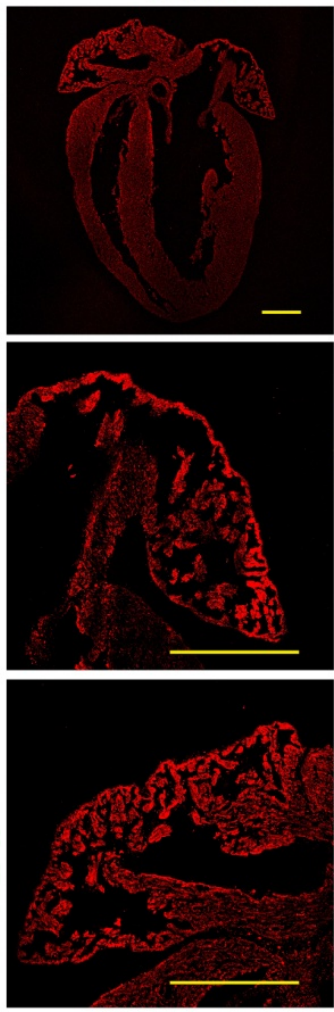

DAPI
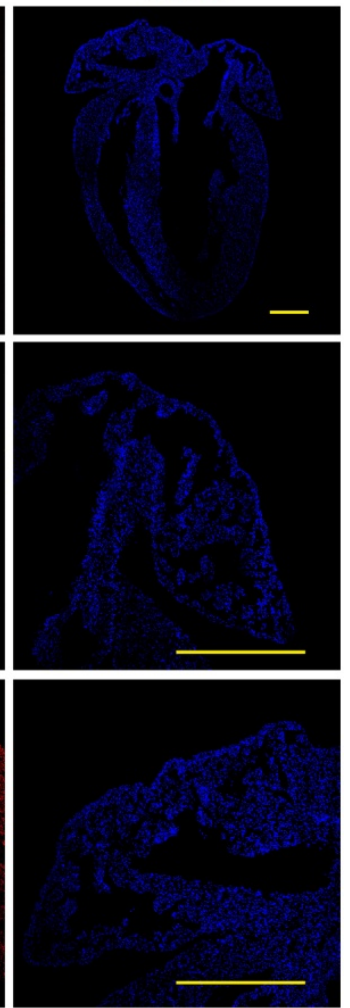

yellow scale bar: $500 \mu \mathrm{m}$

Figure 12. Immunostaining image of P3 Nppa-GFP heart section.

P5. The left and right atria were almost filled with GFP-positive cardiomyocytes and formed a directional, thick bundle of muscle (Figure 13).

P7. The trabecular myocardium evacuated again to form more hollow constructions as the cavity volume increased (Figure 14).

P14. The trabecular myocardium was connected with surrounding cardiomyocytes to form a thick bundled myocardium (Figure 15).

$\mathrm{P} 28$. The fascicular myocardium of the left and right atria continues to thicken. The right atrium predominantly formed a larger cavity structure in the central and rear. While irregular abundant cavities emerged in distinct areas of the left atrium (Figure 16).

P8w. GFP-positive trabecular myocardium in the right atrium embosomed the atrial cavity based on the atrial wall and formed a cavity in the vicinity of the atrial septum. While the left atrium had a grander cavity in the central and posterior part compared to the right. There were also a few slender apertures bordered by the trabecular myocardium and atrial wall (Figure 17).

\section{Non-single foundation origin trabecular cardiomyocytes}

To trace the fate of embryonic trabecular cardiomyocytes, we crossed Nppa2A-CreER with R26-tdTomato mice and collected heart samples at different time points for analysis.

E12.0 - E15.5. Immunostaining was performed on the embryonic heart section. 


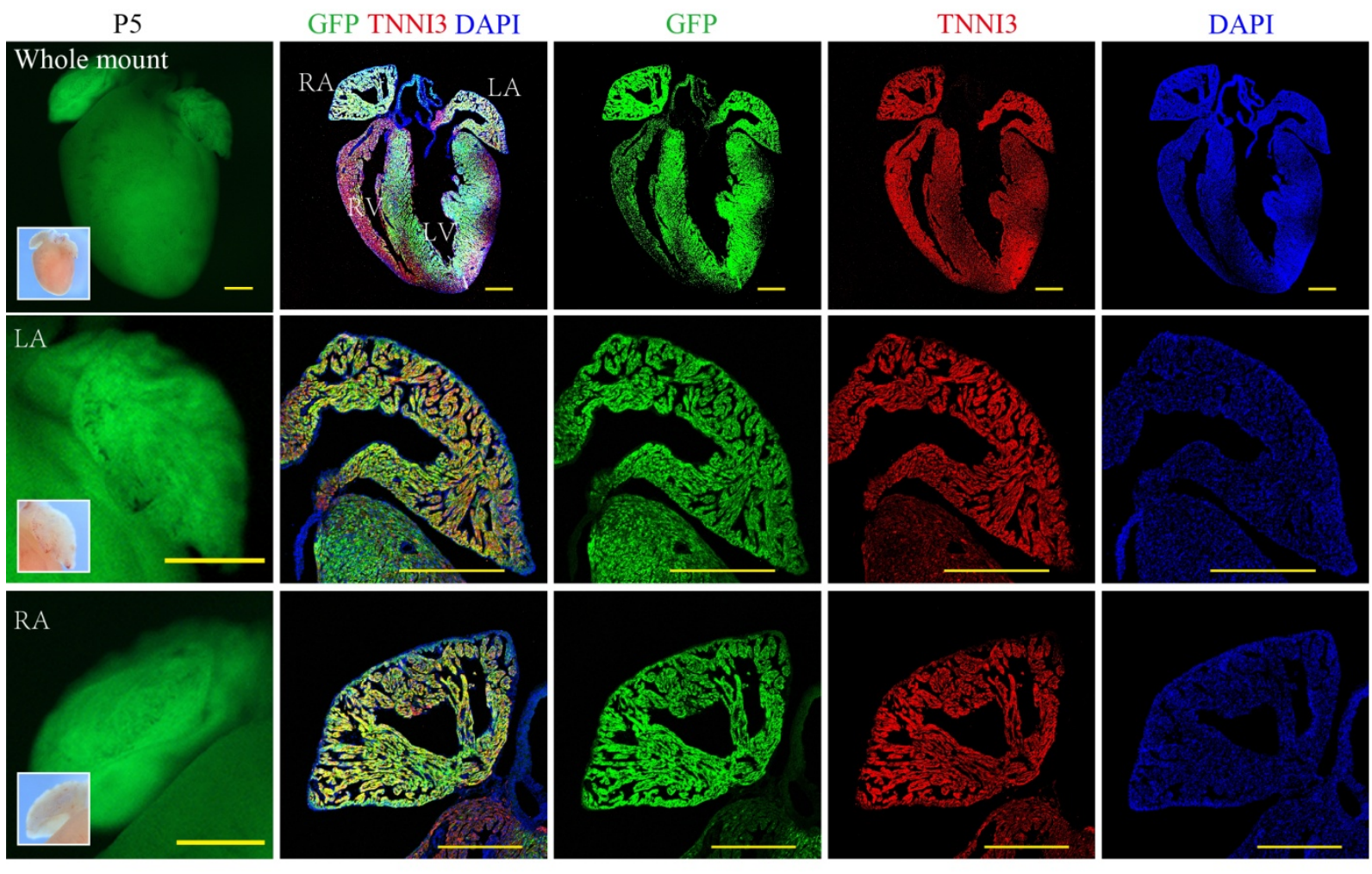

yellow cale bar: $500 \mu \mathrm{m}$

Figure 13. Immunostaining image of P5 Nppa-GFP heart section.

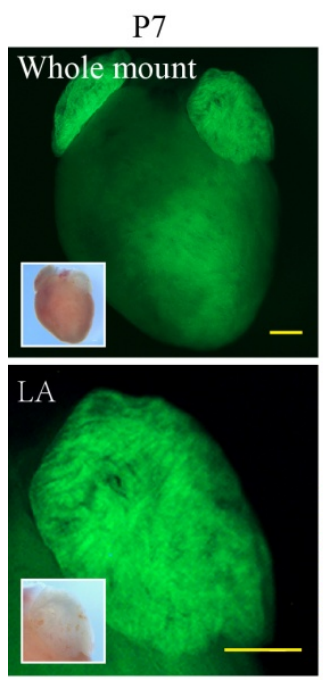

GFP TNNI3 DAPI
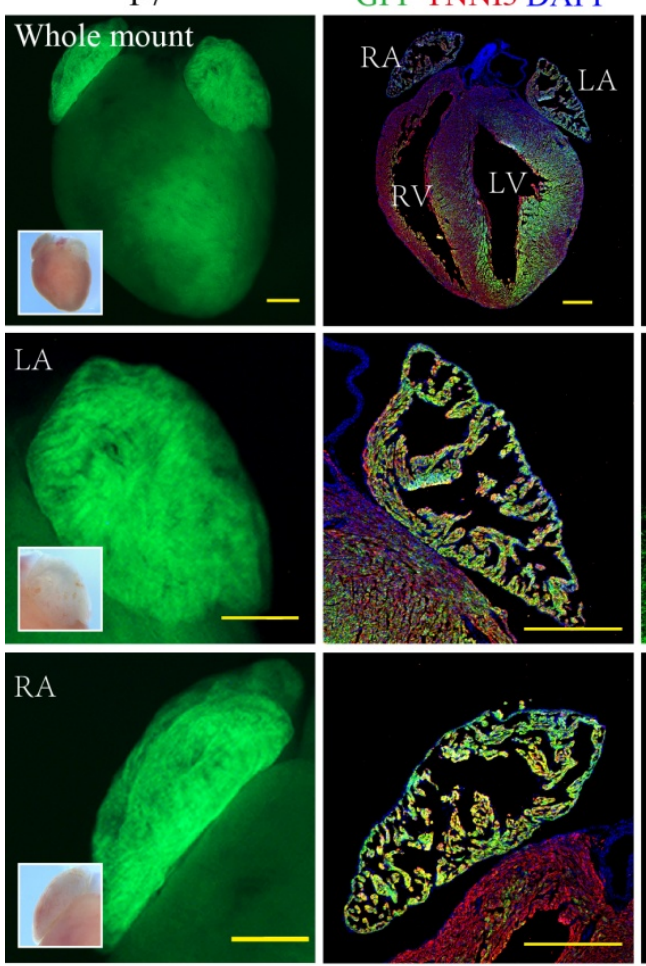

GFP
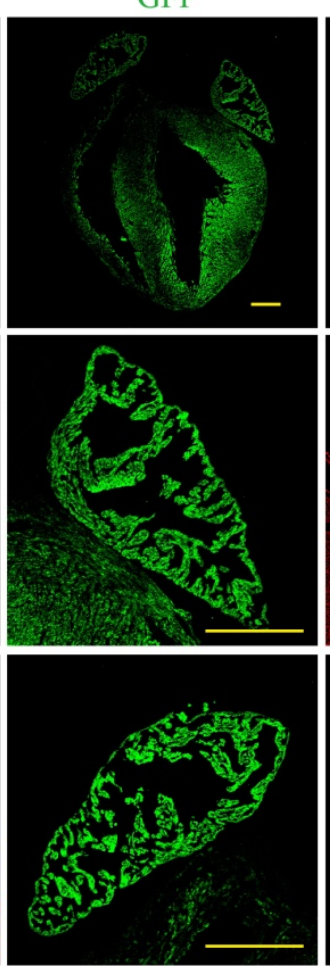

TNNI3
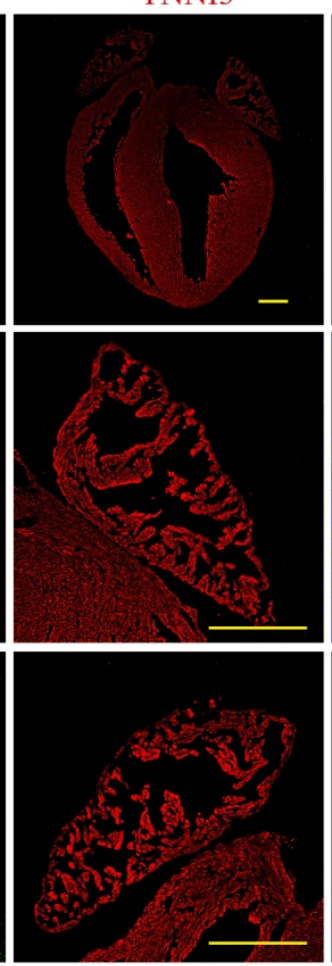

DAPI
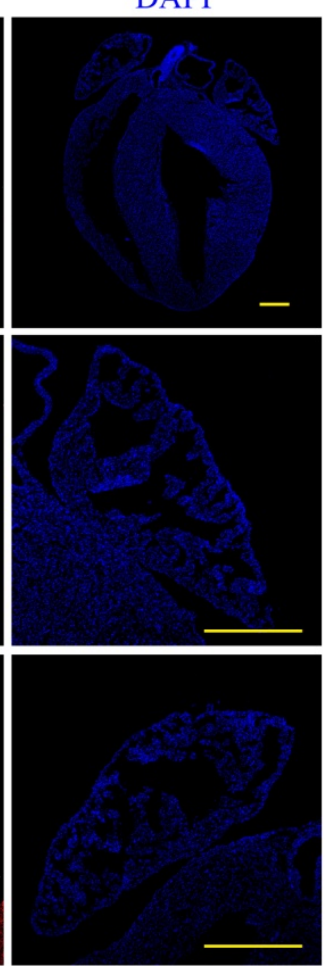

yellow scale bar: $500 \mu \mathrm{m}$

Figure 14. Immunostaining image of P7 Nppa-GFP heart section. 


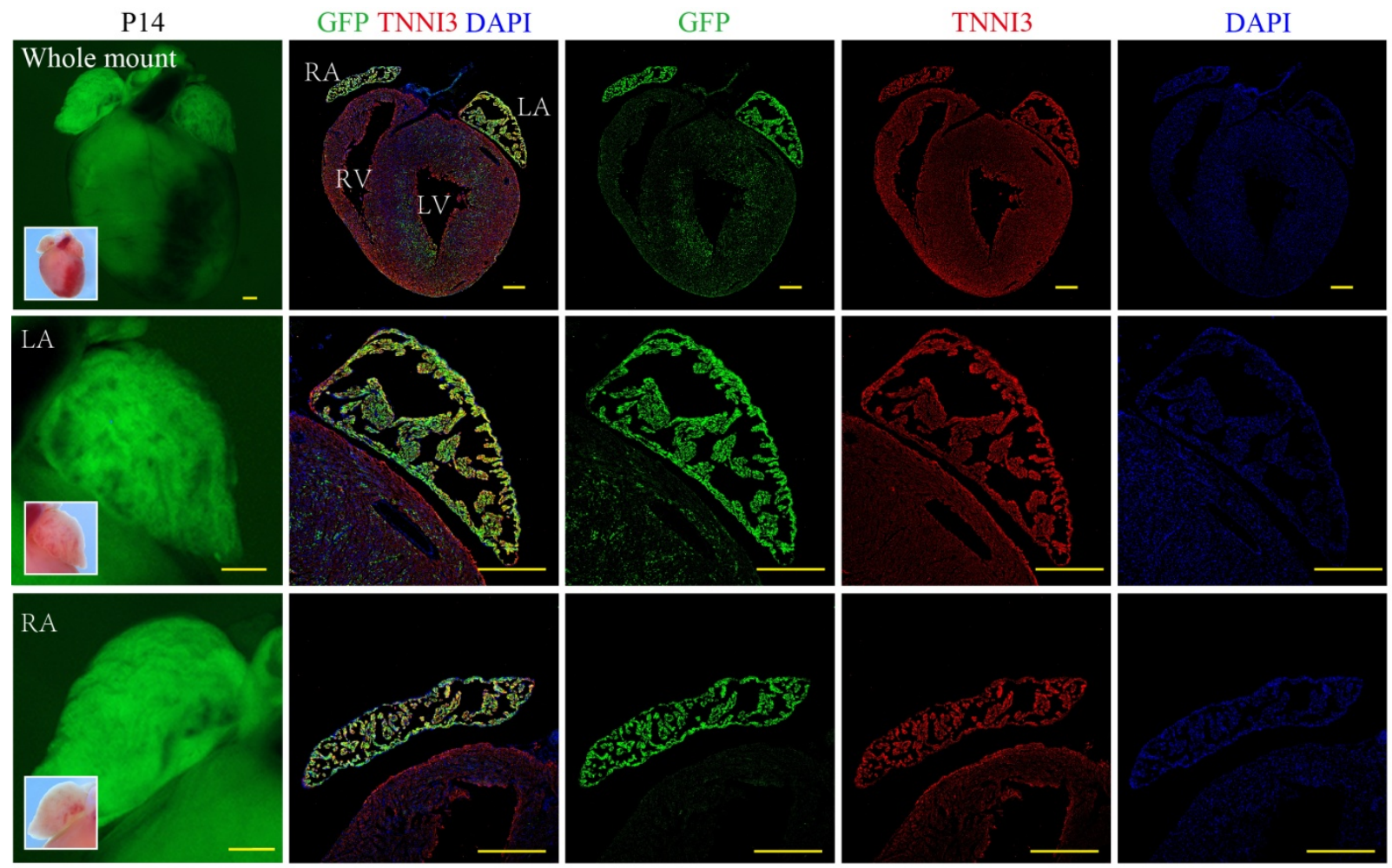

yellow scale bar: $500 \mu \mathrm{m}$

Figure 15. Immunostaining image of P14 Nppa-GFP heart section.

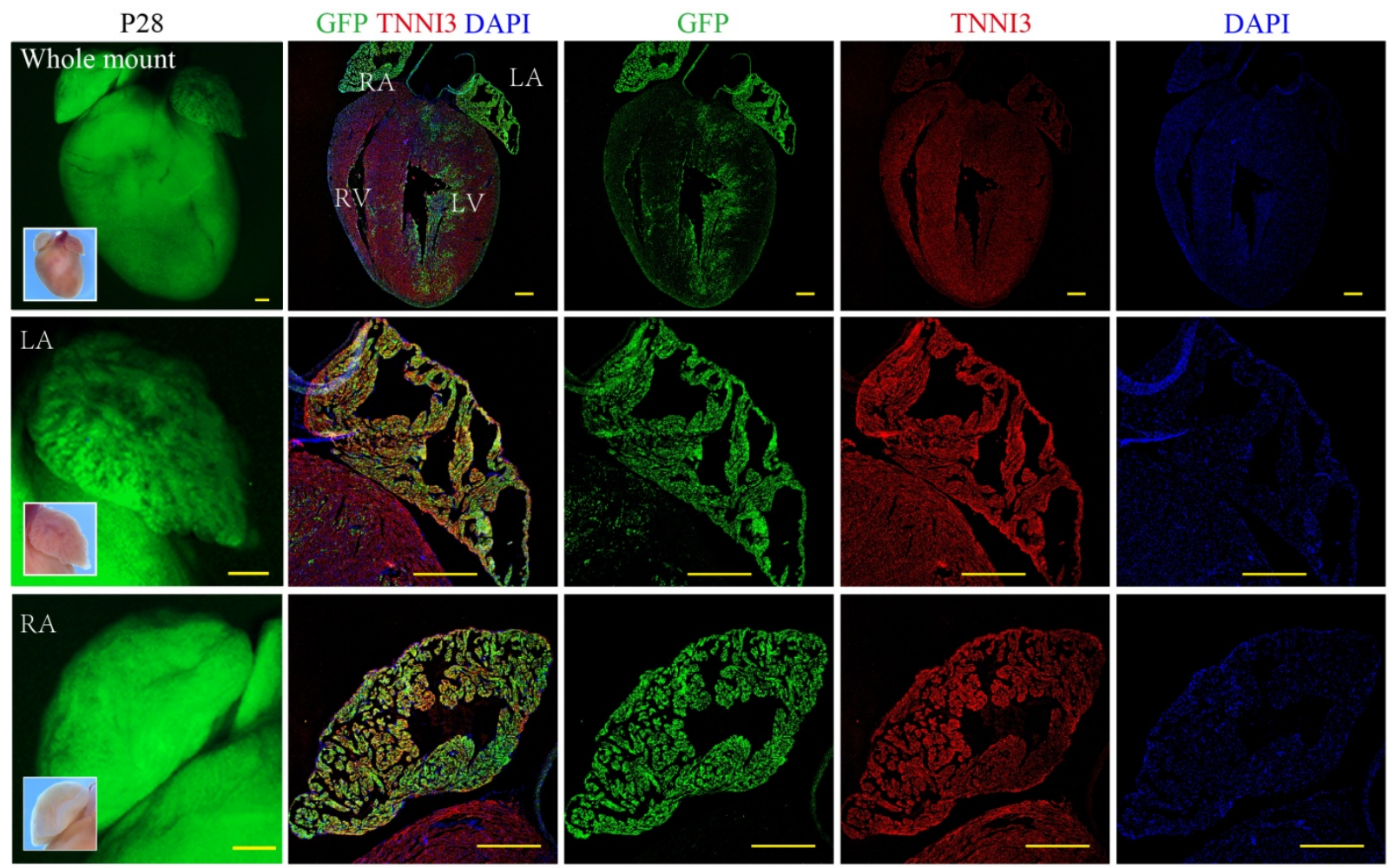

yellow scale bar: $500 \mu \mathrm{m}$

Figure 16. Immunostaining image of P28 Nppa-GFP heart section. 

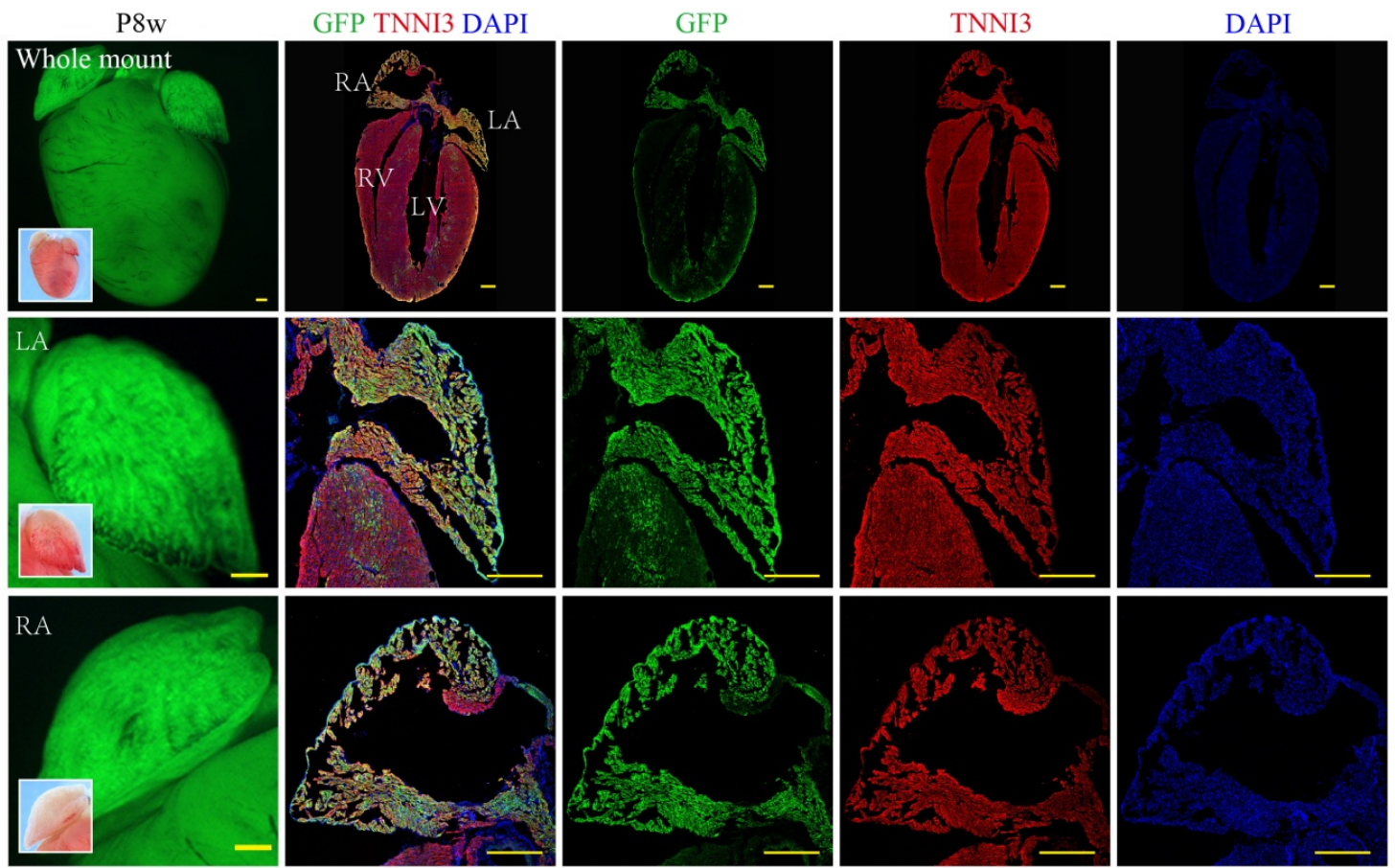

yellow scale bar: $500 \mu \mathrm{m}$

Figure 17. Immunostaining image of P8w Nppa-GFP heart section.
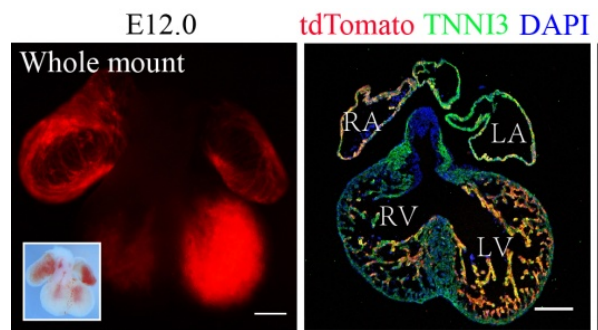

tdTomato
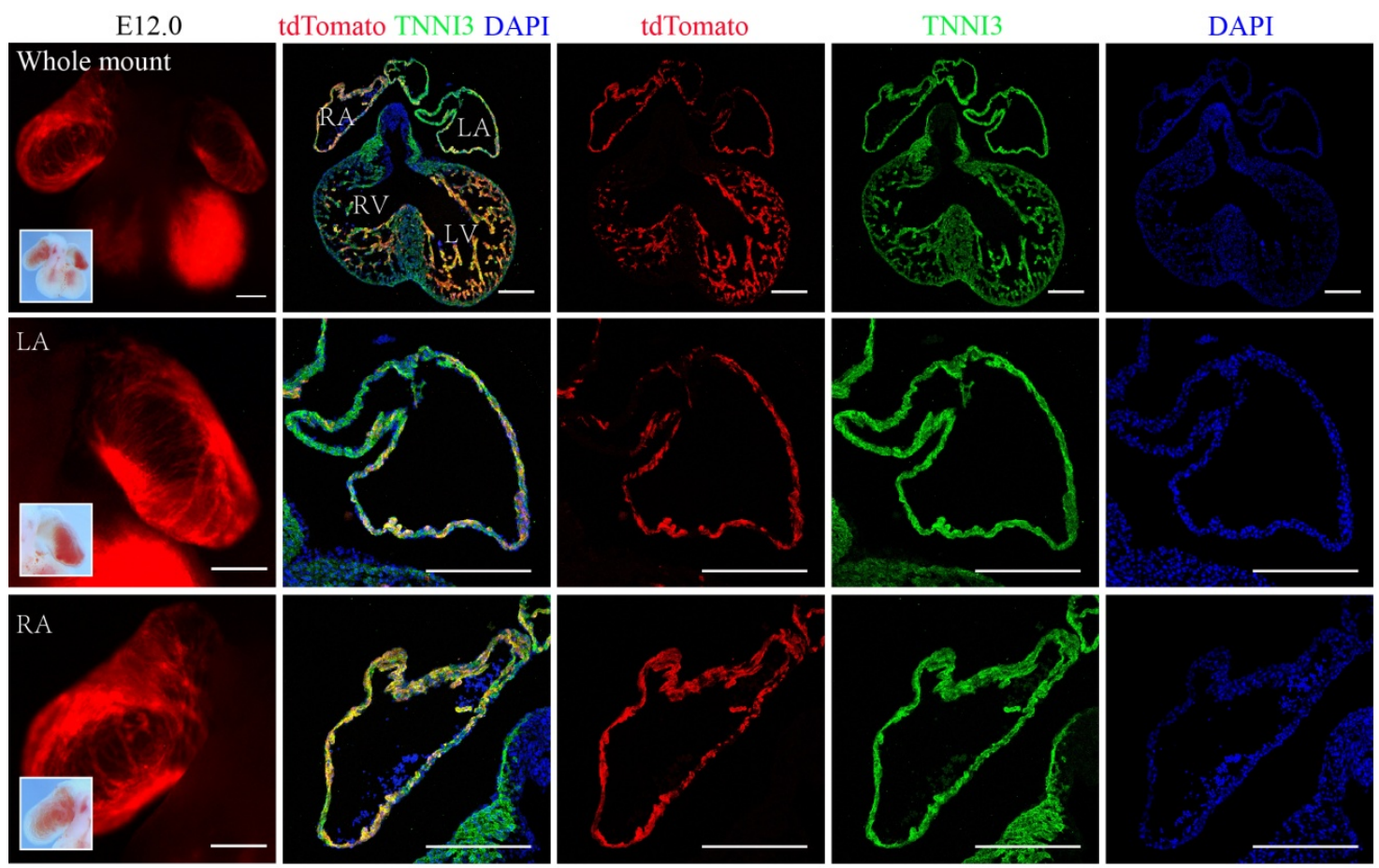

Scale bar: $250 \mu \mathrm{m}$

Figure 18. Fate-mapping of embryonic trabecular cardiomyocytes. Inserts are bright-field images of same hearts or portions. RA, right atria. tdTomato, Alexa Fluor 555. TNNI3, Alexa Fluor 488. DAPI, nuclear staining. LA, left atria. RV, right ventricle. LV, left ventricle. Scale bars are indicated. For brevity and clarity, the following images Figures 19-25 will no longer be annotated as they are similar in structure except the timepoint, and the abbreviations below have the same meaning. 


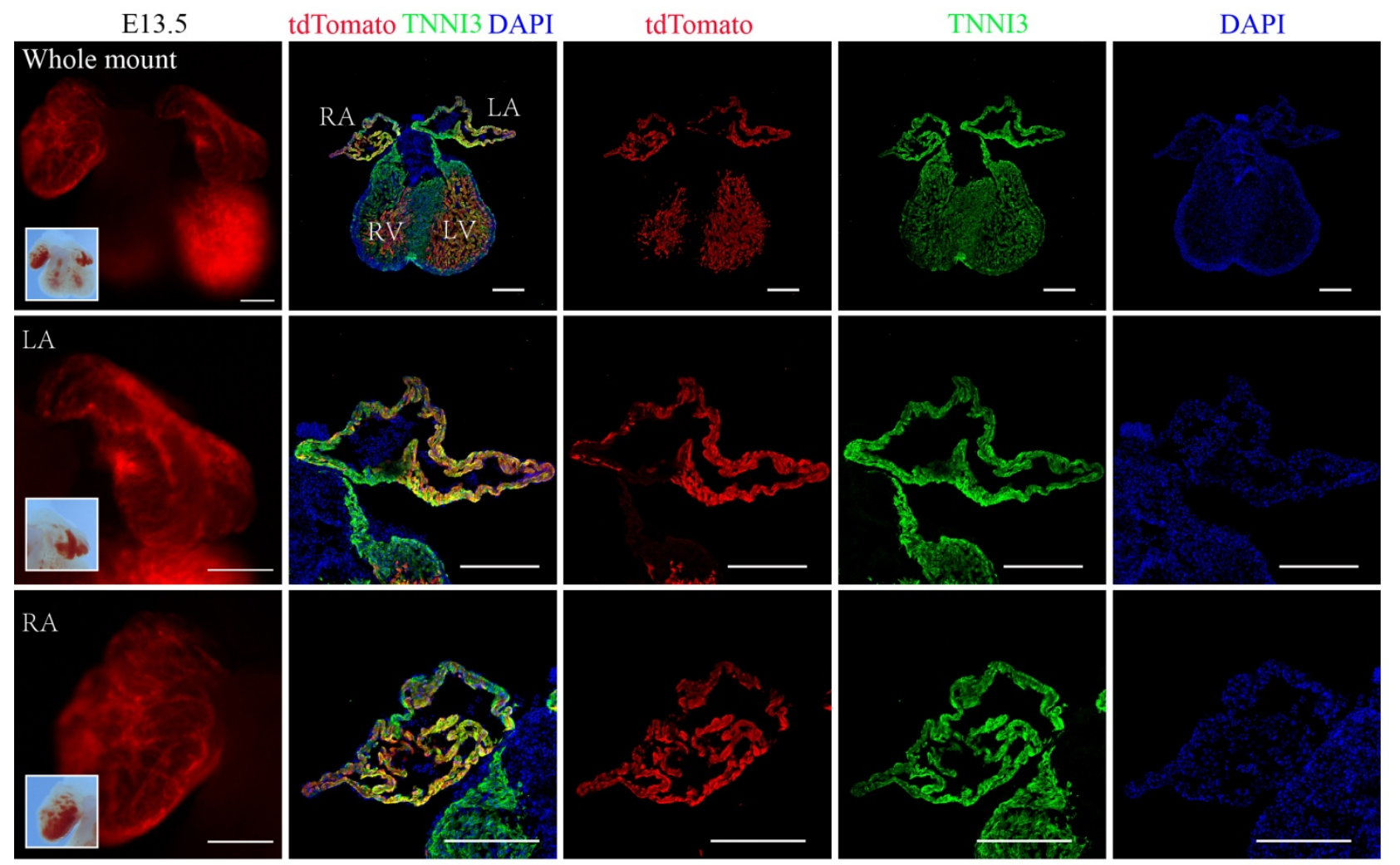

E14.5

tdTomato
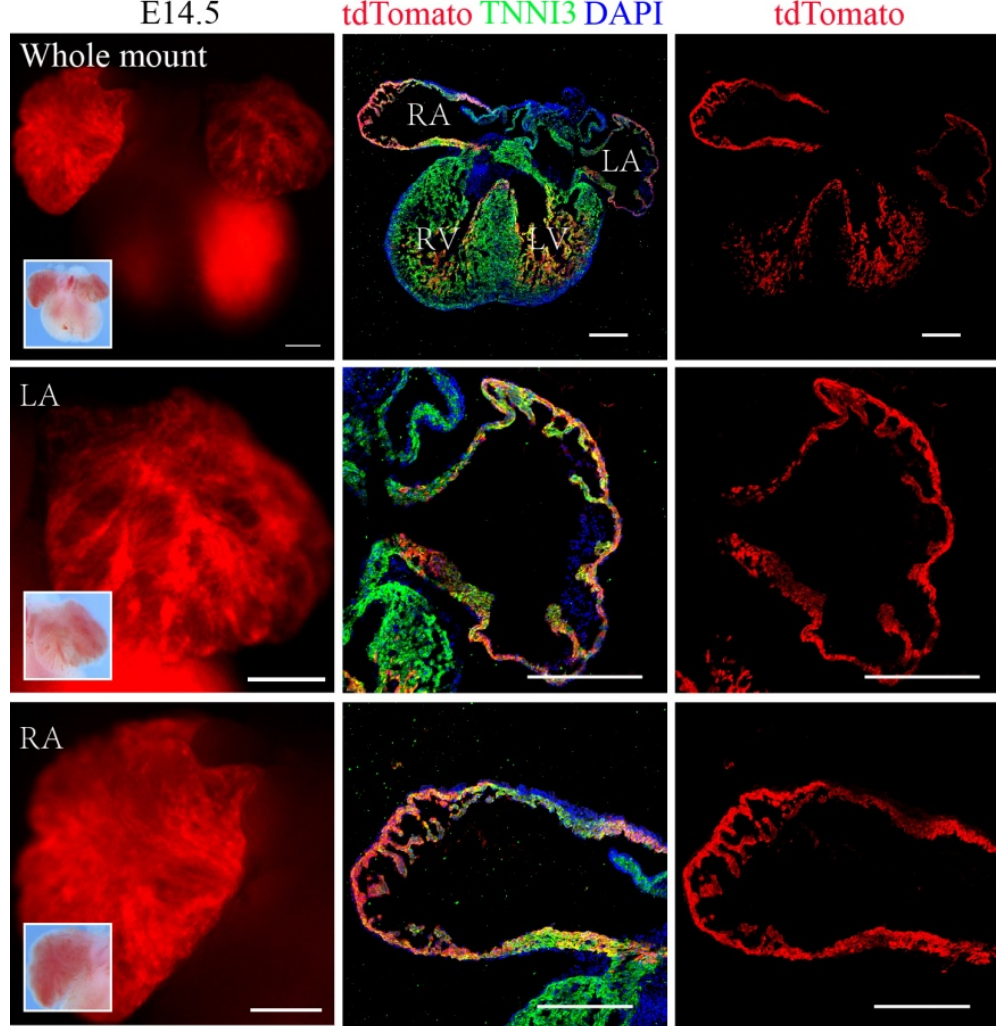

TNNI3
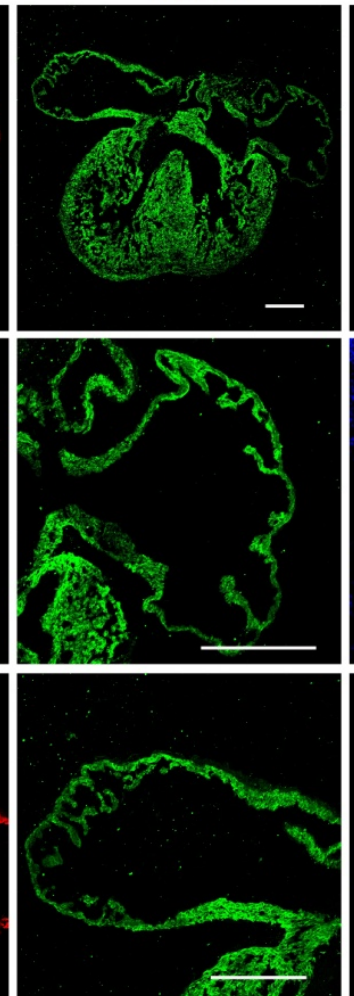

Scale bar: $250 \mu \mathrm{m}$ DAPI

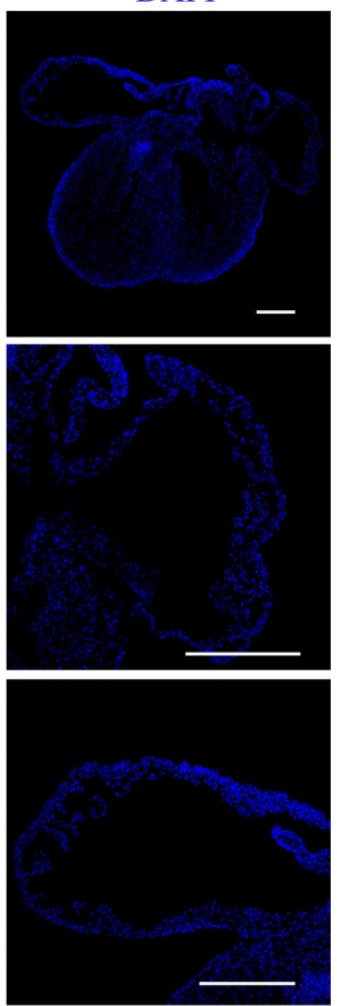

Scale bar: $250 \mu \mathrm{m}$

Figure 19. Immunostaining image of E13.5 and E14.5 Nppa-CreER; tdTomato heart section. 


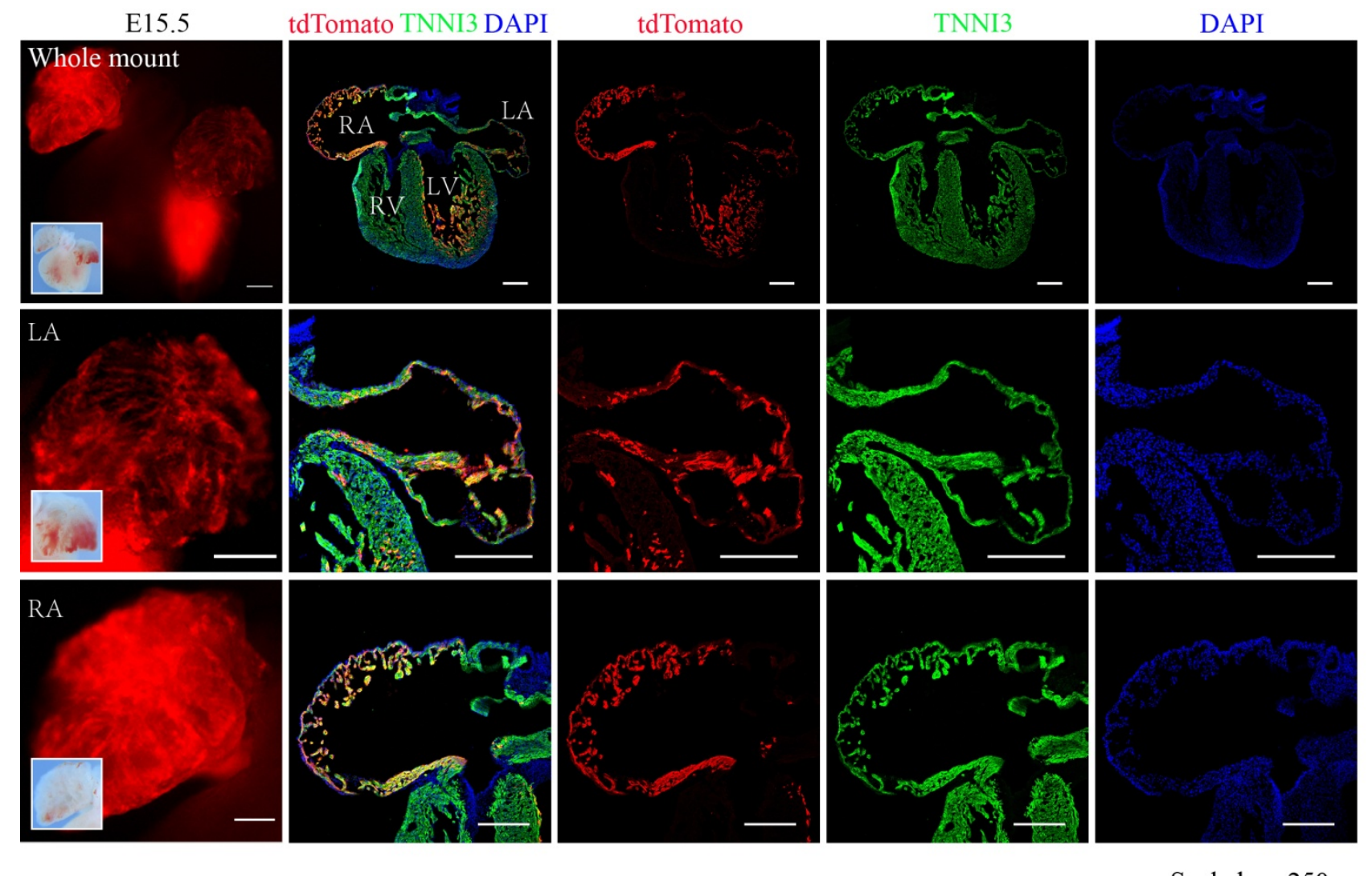

Scale bar: $250 \mu \mathrm{m}$

Figure 20. Immunostaining image of E15.5 Nppa-CreER; tdTomato heart section.

With the detection of tdTomato positive cells, we could trace the endogenous Nppa-positive cells. In the left and right atrium, we detected tdTomato positive cells in myocardial walls and myocardial trabeculae, which overlapped with the myocardial specific signal TNNI3. The fluorescent protein expression pattern of the right atrium was slightly different from the left atrium. The overall situation was that the number of tdTomato-positive cells in the right atrium was significantly larger than that in the left atrium. However, there were also common points that the ratio of tdTomato-positive cells in the upper wall of the left and right atria to the number of cardiomyocytes was higher than the inferior wall (myocardial wall at the bottom of the atrium) (Figures 18-20).

E16.5 - E17.5. As the development progressed and the volume of the atrial cavity augmented, the number of tdTomato-positive cardiomyocytes continuously increased, restricted both in the atrial myocardial wall and myocardial trabeculae; what remained unchanged was the number of tdTomato-positive myocardial cells in the right atrium was still significantly higher than that of the left atrium, and the trabecular myocardium populations in the middle, posterior and top of the right atrium showed significantly fewer tdTomato-positive cardiomyocytes. The anterior part of the left atrium and the posterior part close to the atrial septum appeared similar results. This indicated that the source of the trabecular myocardium protruding into the heart cavity was not a single one, or that the 


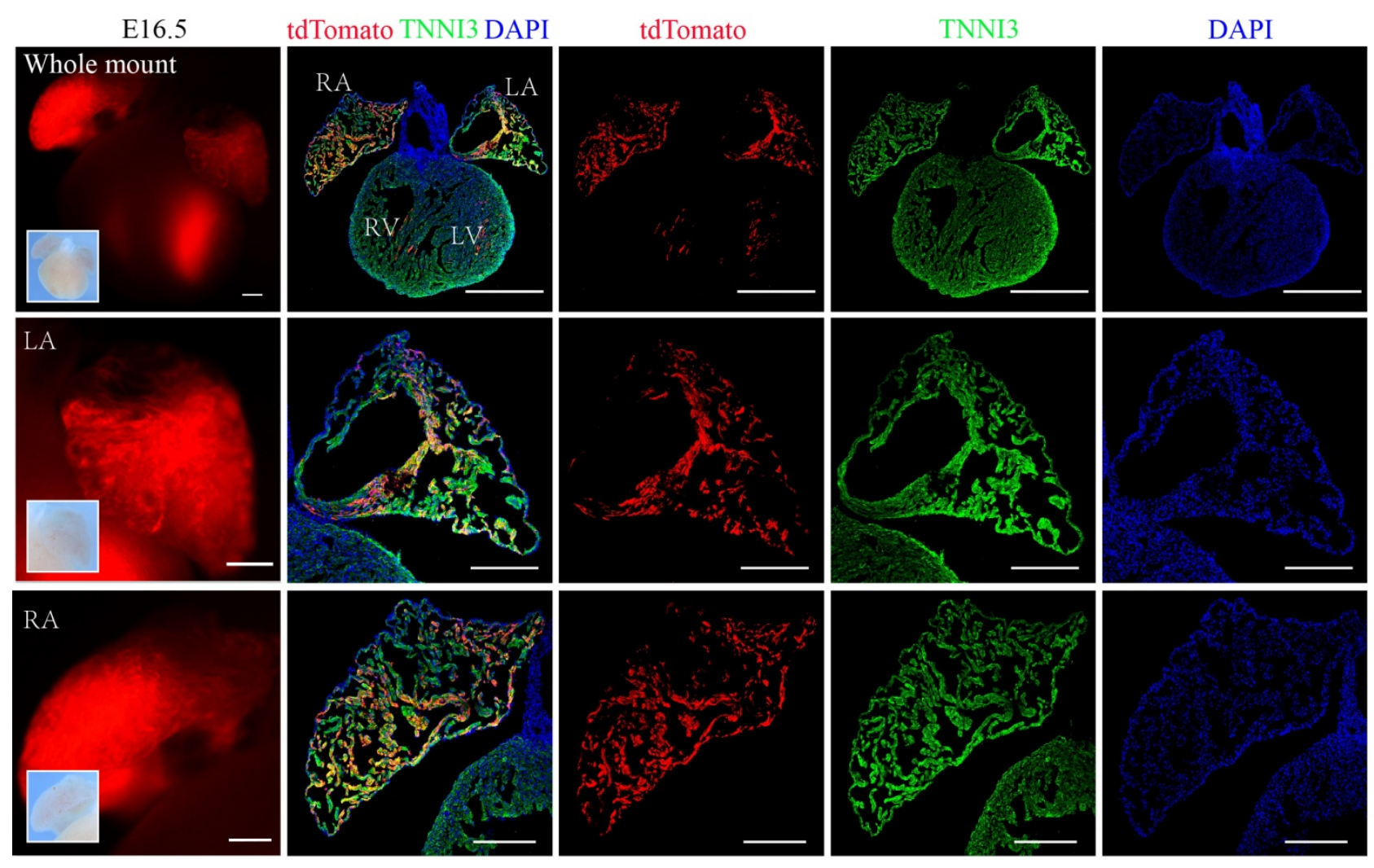

E17.5
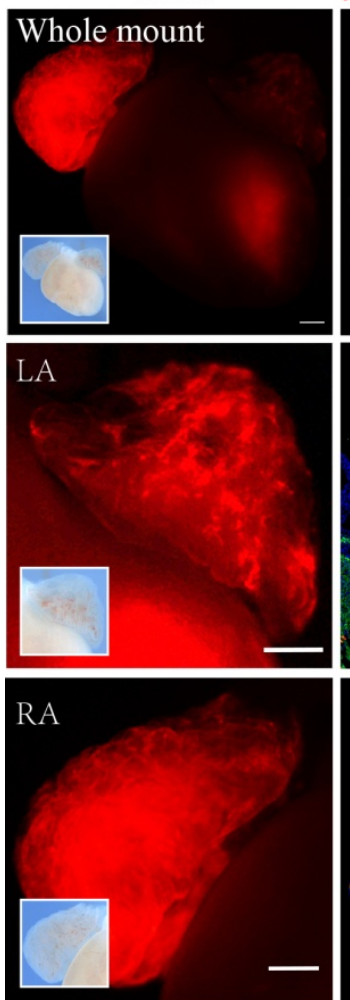

dTomato TNNI3 DAPI
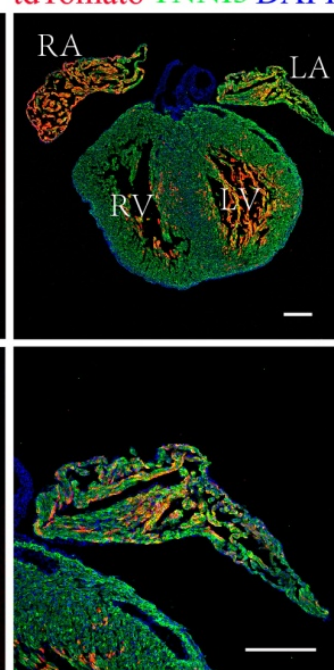

$-$

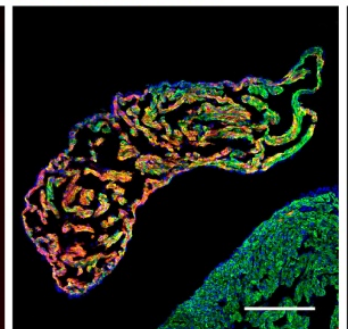

tdTomato

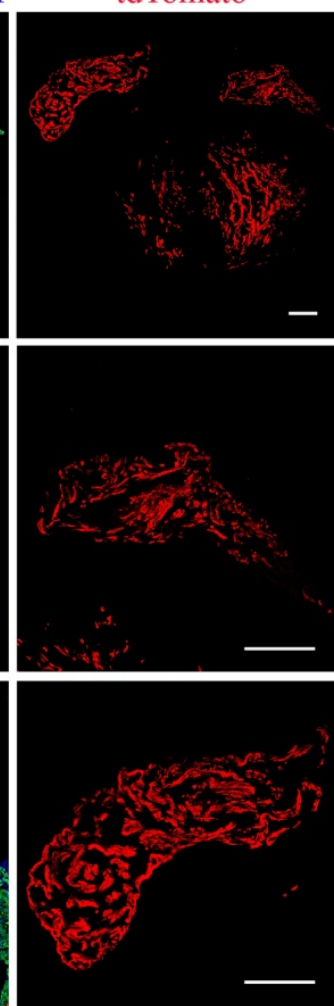

Scale bar: $250 \mu \mathrm{m}$ DAPI

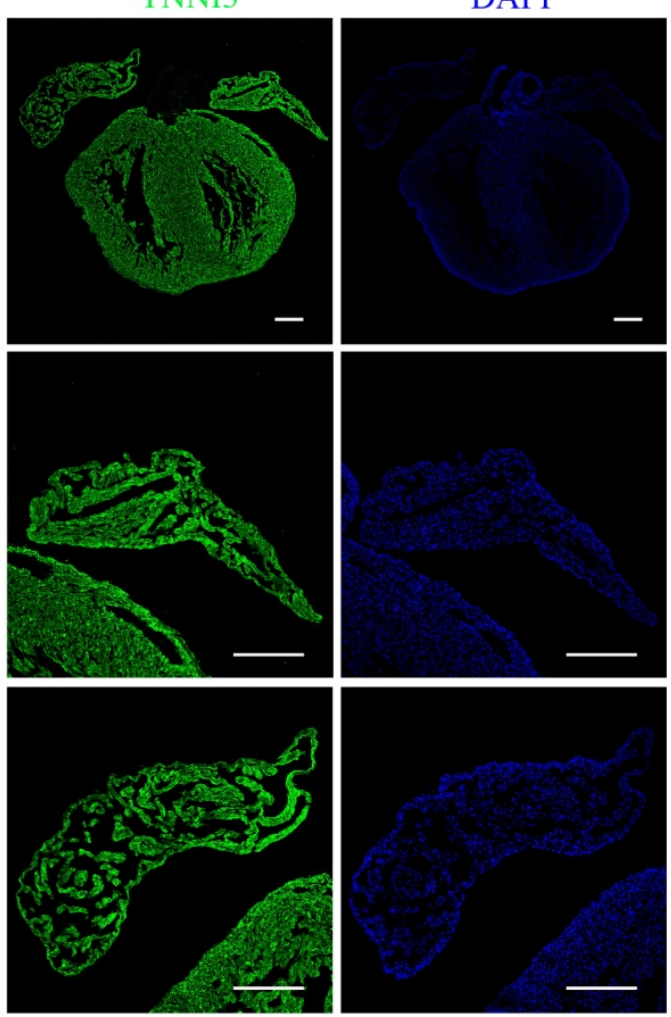

Scale bar: $250 \mu \mathrm{m}$

Figure 21. Immunostaining image of E16.5 and E17.5 Nppa-CreER; tdTomato heart section. 
trabecular myocardial cells in different parts of the atrium had different foundations; However, it might also be due to the difference in the time of administration of Tamoxifen or the different induction efficiency of the individual samples (Figure 21).

E18.5 - P0. The reticular trabecular myocardium and cell populations of the myocardial wall in the left and right atriums were all positive for tdTomato, which indicated that the trabecular myocardium protruded into the atrial cavity was derived from the early Nppa-positive cardiomyocyte population (Figure 22).

P3, P5, P7, P14, P28, P8w. Different from the E18.5 - P0 period, for the postnatal heart, there were considerably more tdTomato positive cells in the right atrium than in the left atrium, and tdTomato positive cells were mainly distributed in the lateral and middle of the right atrium. Trabecular myocardium at the top of the atrium was less identified while the tdTomato positive cells in the left atrium were mainly scattered in the central but less at the anterior and posterior top. This phenotype was similar to that at E16.5-E17.5, which indicated that the source of trabecular myocardium protruded into the heart cavity was not a single origin (Figures 23-25).

\section{Discussion}

Arrhythmia such as atrial fibrillation (AF) affecting millions of individuals worldwide, which is characterized by very rapid and uncoordinated atrial activity, and the process and causes of AF can be linked to trigger and substrate. Cellular mechanism of atria development contributes to substrate. Tissue architecture, specifically myocardial framework is the key concept underlying development of AF.

The spatiotemporal expression pattern of Nppa in atrial cardiomyocytes in this article showed a relatively complete and dynamic process of atrial development. We used Nppa positive cardiomyocytes to trace the fate of trabeculae in atria, and they were represented in postnatal trabecular myocardial components. In addition, Nppa positive cardiomyocytes labeled at E11.0 - E12.0 protruded into atrial cavity and during subsequent development to form Nppa-positive myocardial architectures. Atrial wall cardiomyocytes retain contacts with laterally expanding muscle and these cardiomyocytes cloned to form pectinate muscle branches in atrial chmber. The lineage tracing of Nppa-positive cardiomyocytes further revealed the non-single cellular origin of the atrial myocardium. We provided reliable evidence that, with the expansion and coalescence of atrial trabeculae, the hybrid postnatal myocardium zone in the atrium developed. These data suggested that abnormalities in myocardial structure and function resulting from complicated diverse cell or tissue population. Precise mechanism of atrial pathology remains further defined.

Recent reports indicated that the left atrial appendage was closely related to thrombosis and arrhythmia [10] [11]. More recent efforts have focused on identifying the underlying cellular mechanism that lead to atrial remodeling [12]. In 

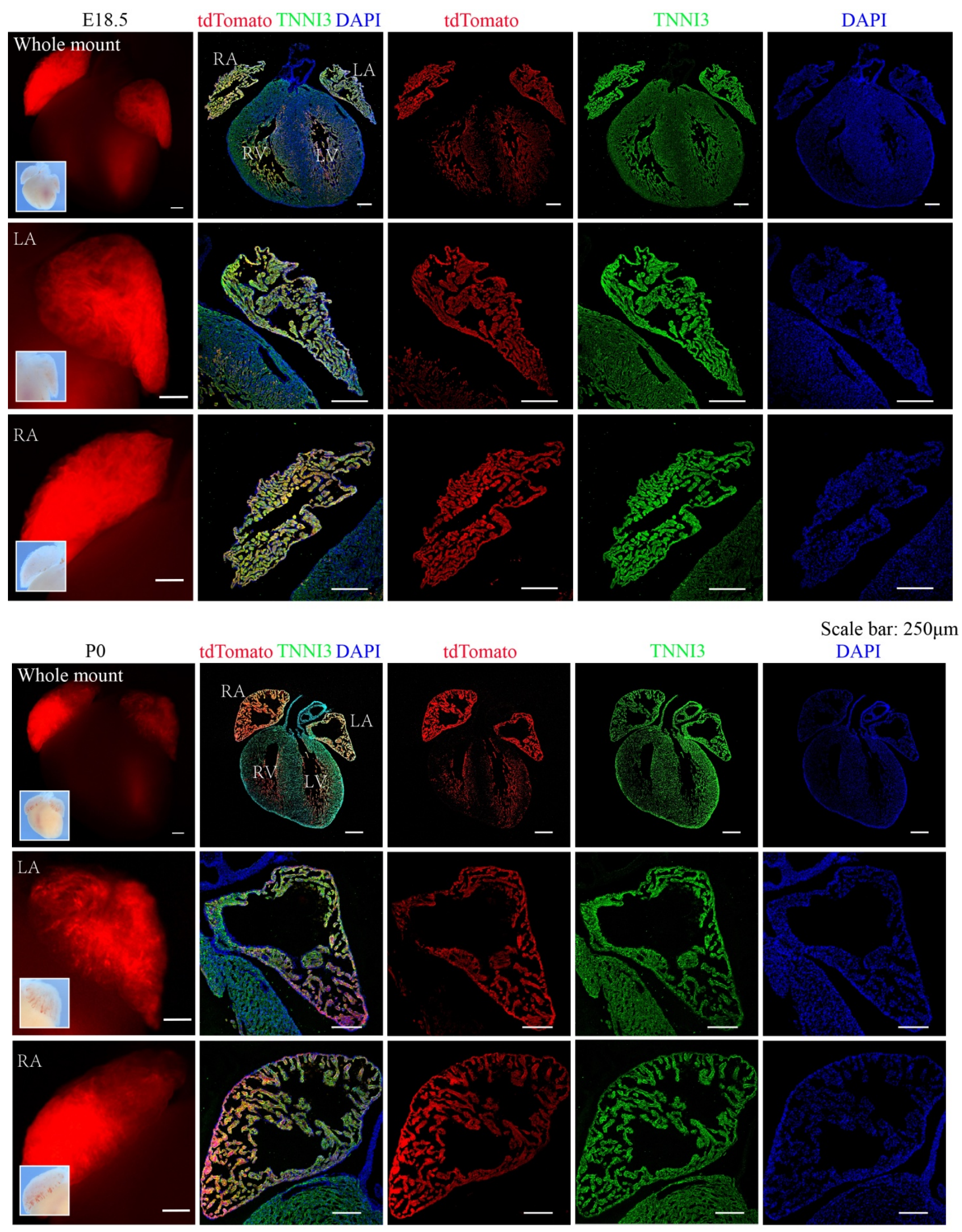

Scale bar: $250 \mu \mathrm{m}$

Figure 22. Immunostaining image of E18.5 and P0 Nppa-CreER; tdTomato heart section. 


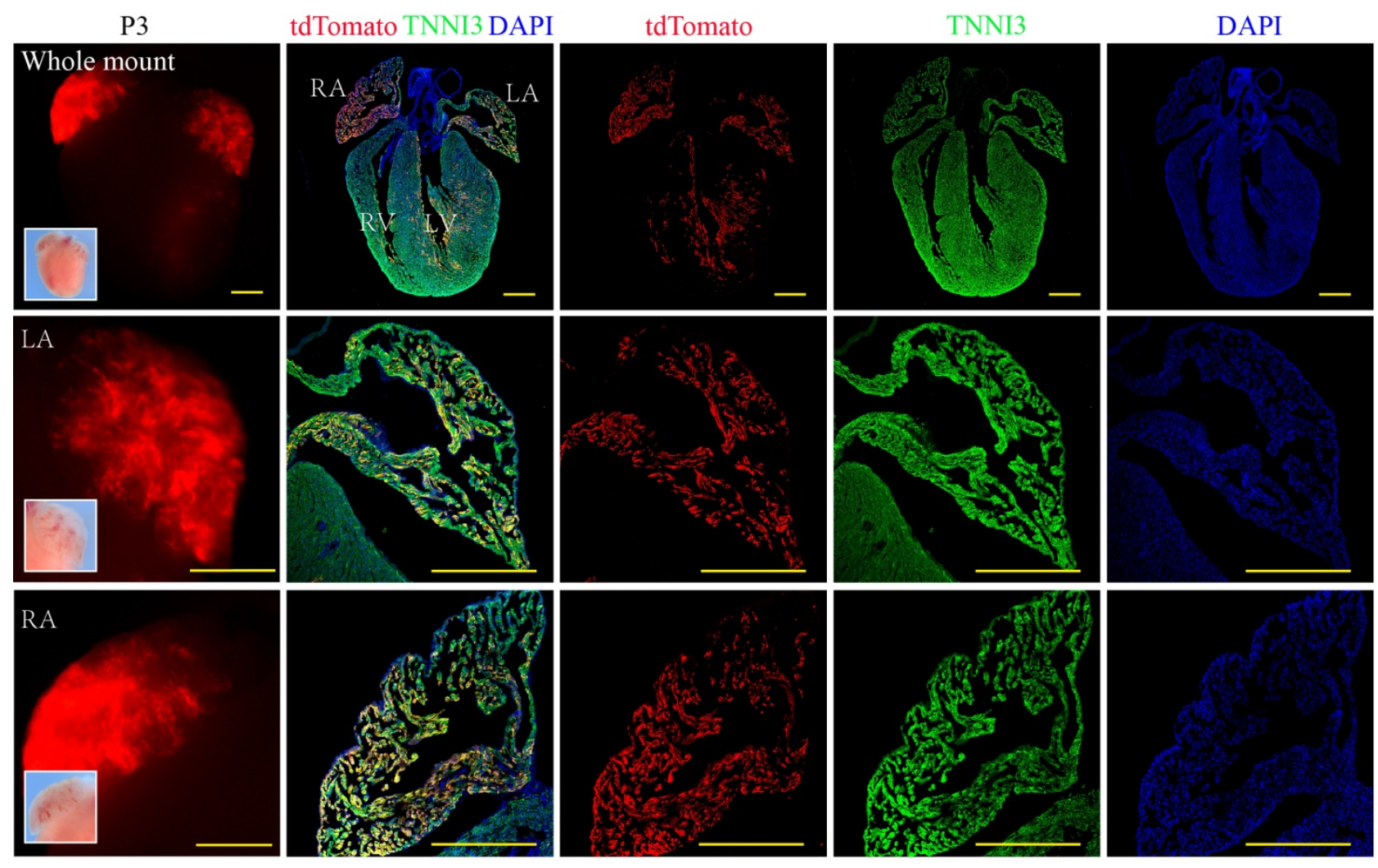

P5
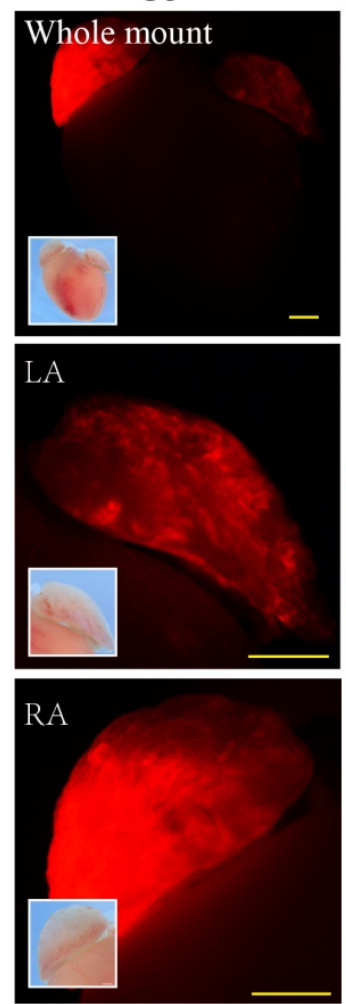

tdTomato TNNI3 DAPI
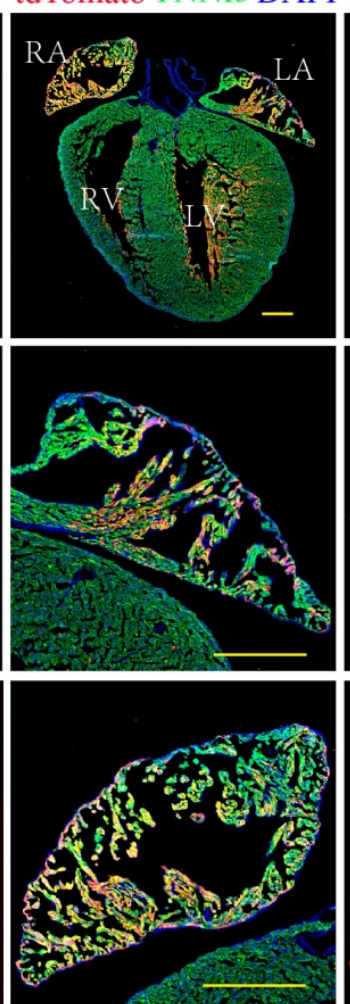

tdTomato
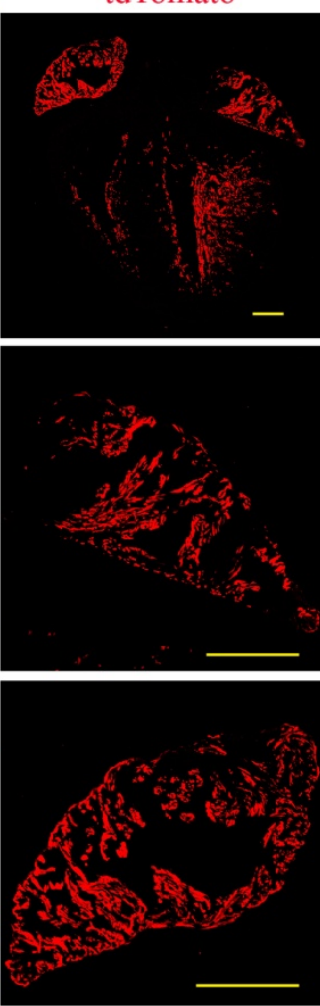

yellow scale bar: $500 \mu \mathrm{m}$

TNNI3
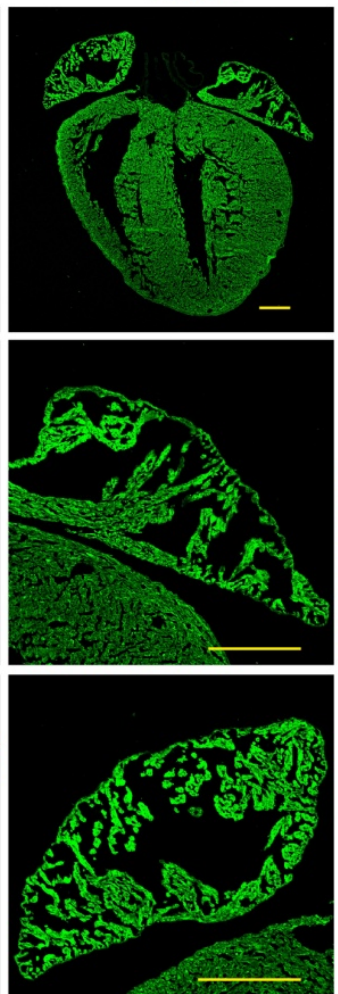

DAPI

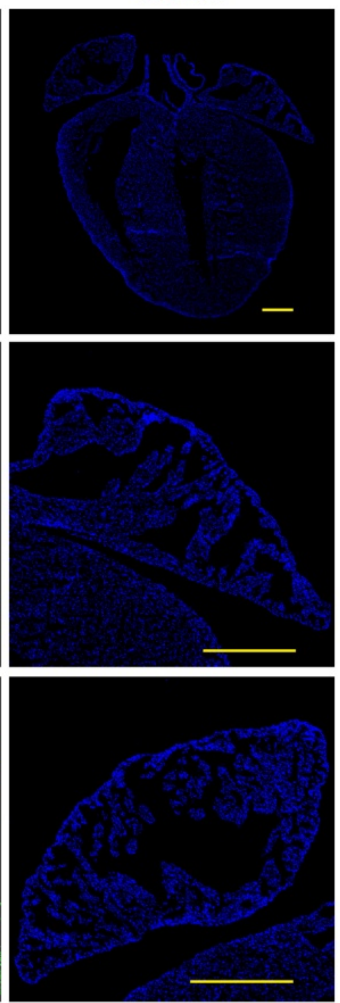

yellow scale bar: $500 \mu \mathrm{m}$

Figure 23. Immunostaining image of P3 and P5 Nppa-CreER; tdTomato heart section. 


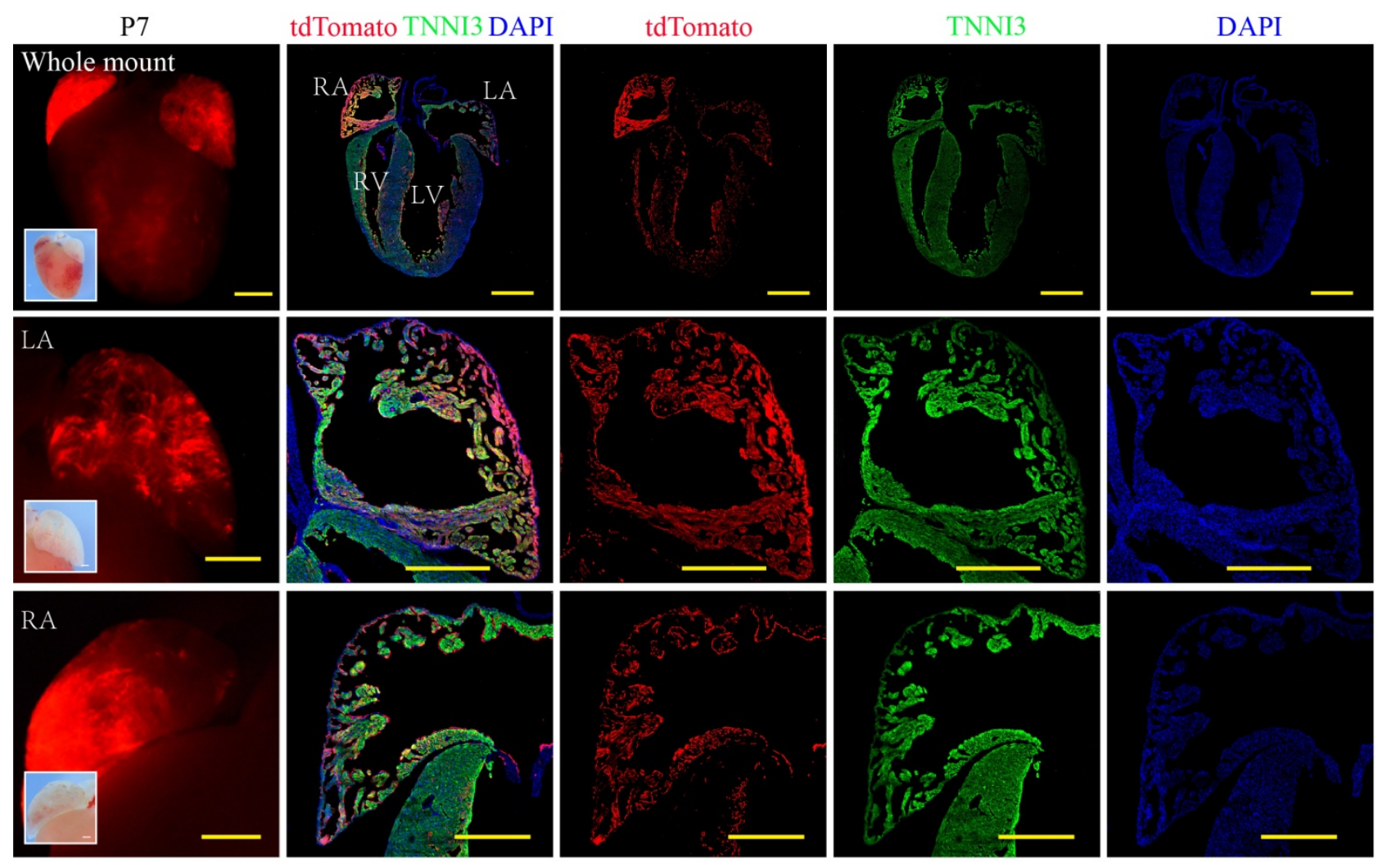

P14

tdTomato TNNI3 DAPI

tdTomato

yellow scale bar: $500 \mu \mathrm{m}$
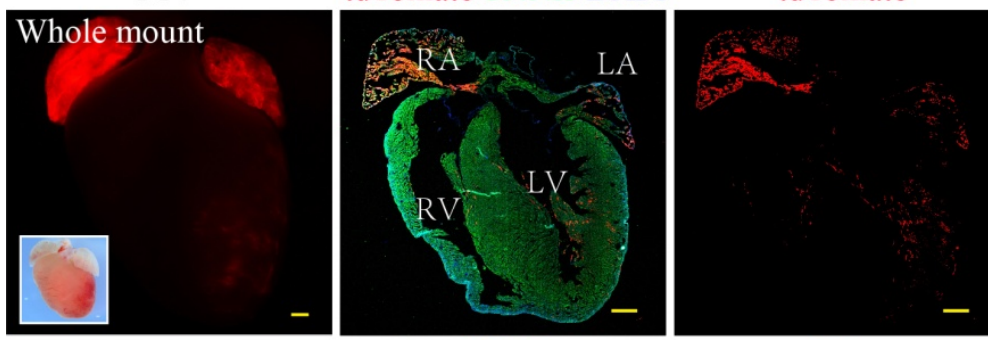

TNNI3

DAPI
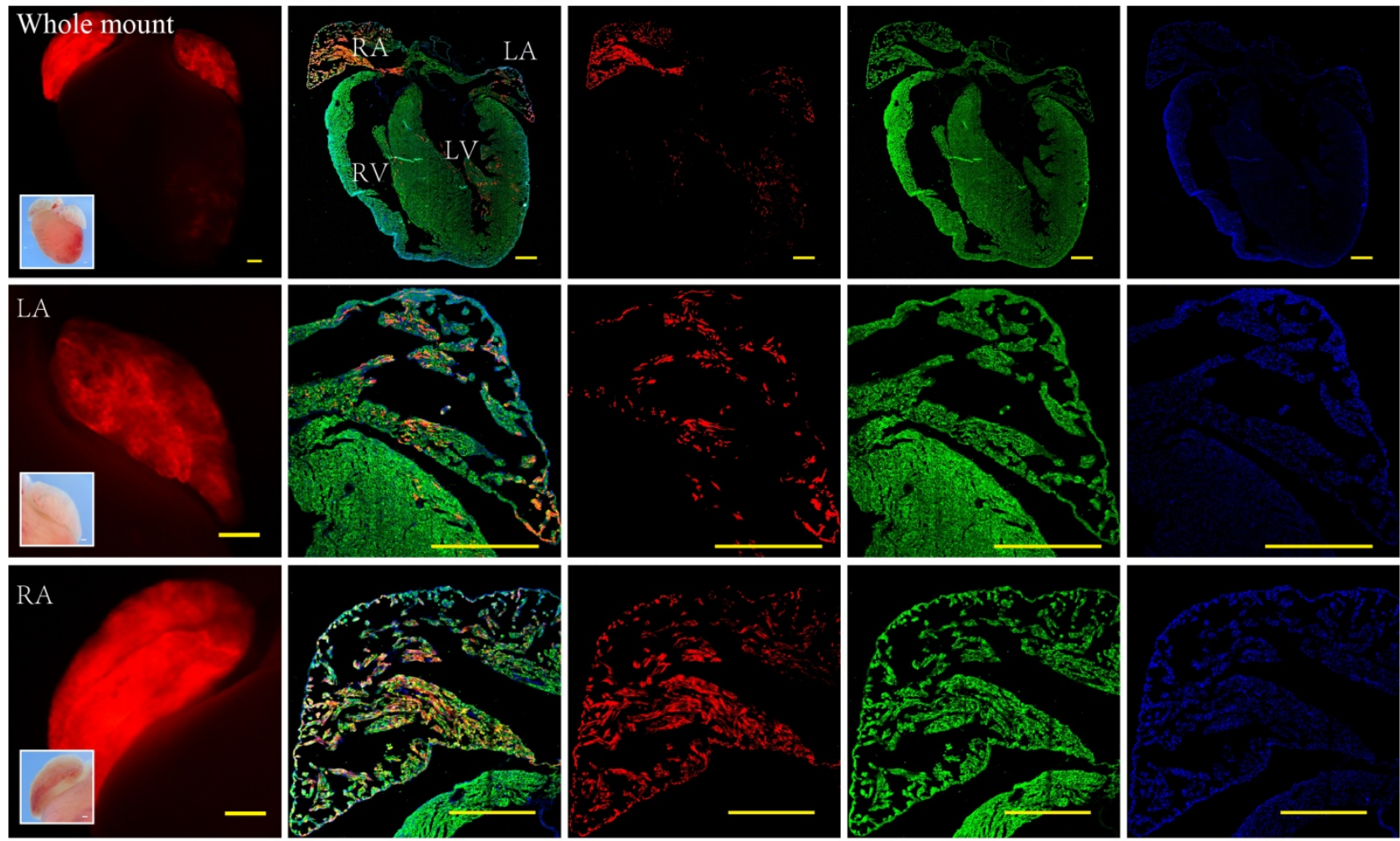

yellow scale bar: $500 \mu \mathrm{m}$

Figure 24. Immunostaining image of P7 and P14 Nppa-CreER; tdTomato heart section. 


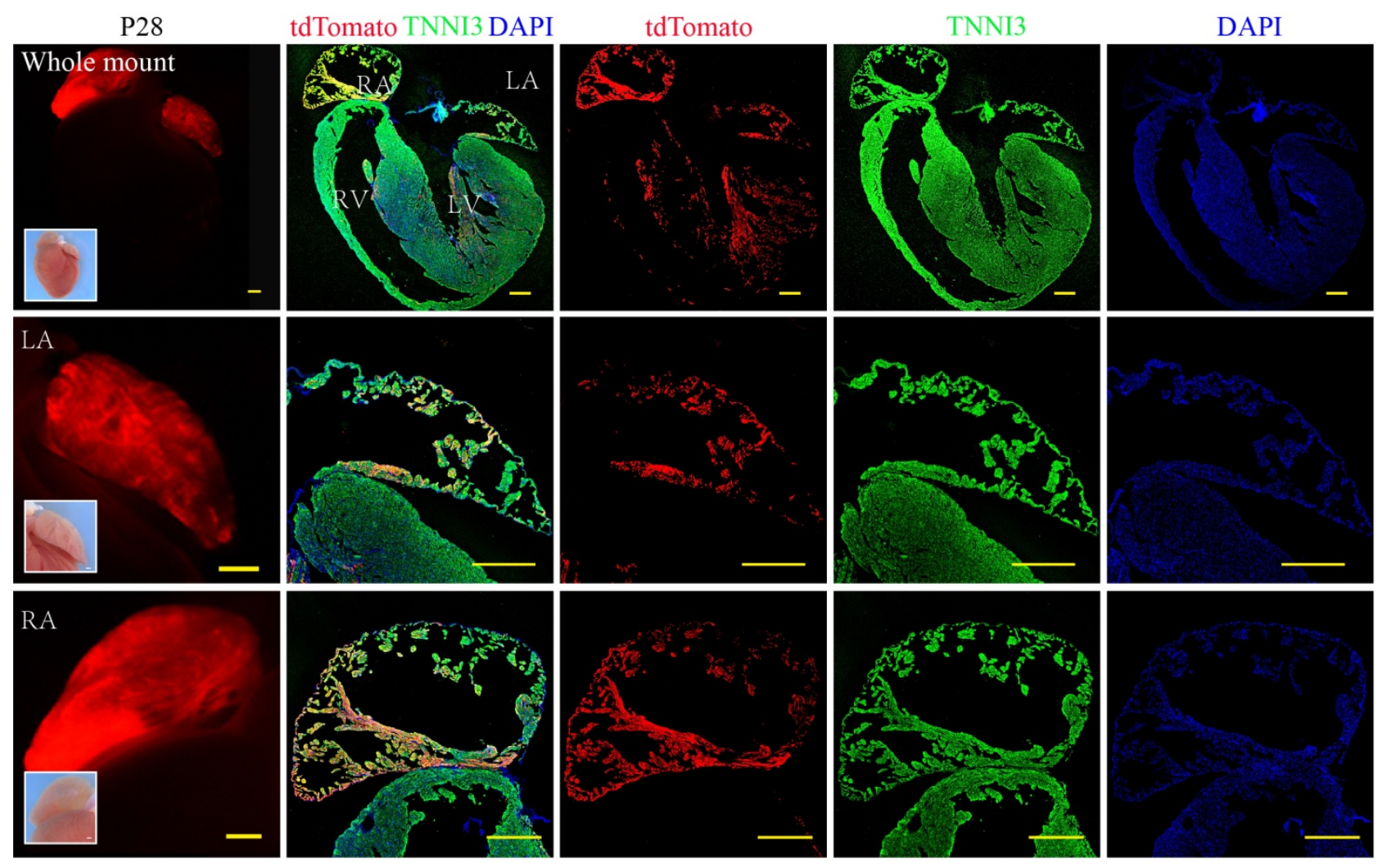

P8w

tdTomato TNNI3 DAPI

tdTomato

yellow scale bar: $500 \mu \mathrm{m}$
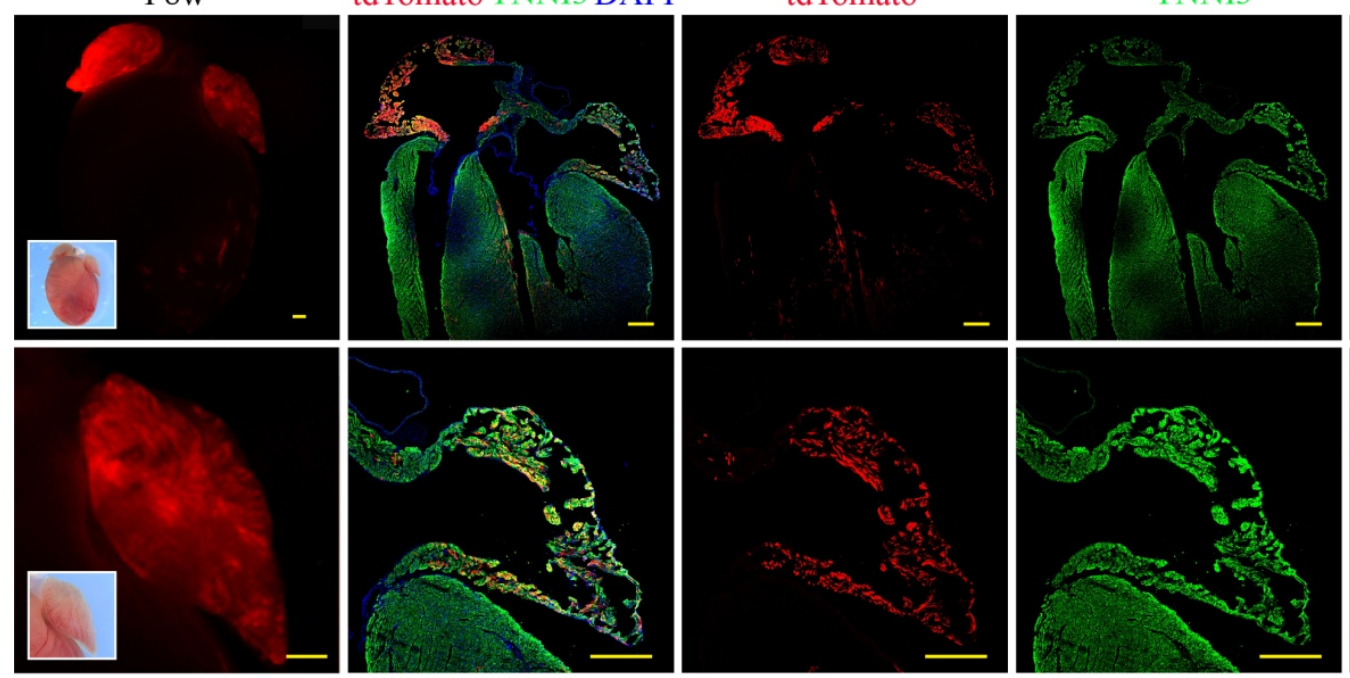

DAPI
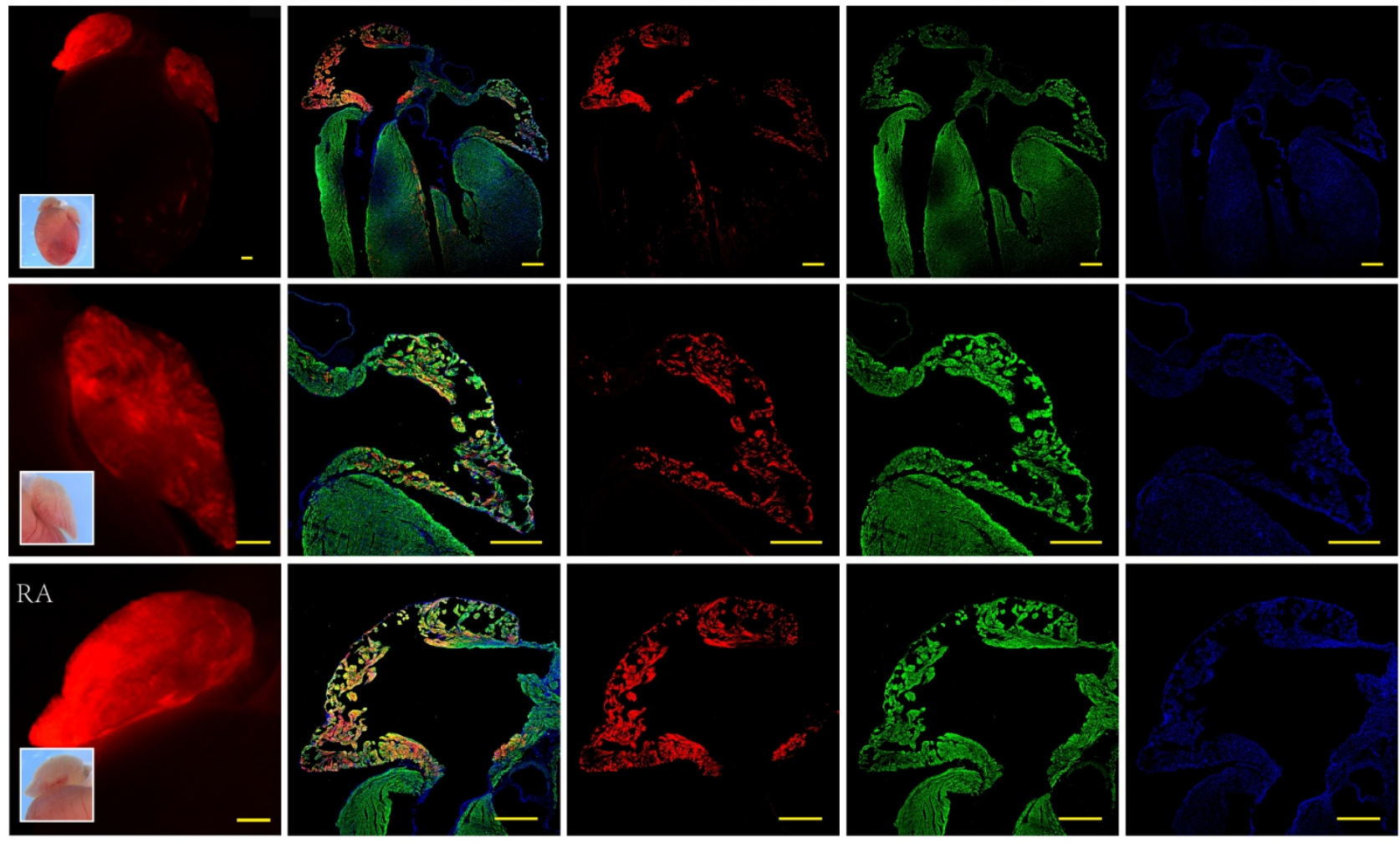

yellow scale bar: $500 \mu \mathrm{m}$

Figure 25. Immunostaining image of P28 and P8w Nppa-CreER; tdTomato heart section. 
clinical research, advanced structural atrial remodeling has been difficult to process. Further mechanical studies are needed to improve understanding arrhythmia such as AF. In the follow-up, we may also focus on the research on the cellular mechanism of the development of the left and right atrial appendages, which is of great significance for revealing the causes of heart diseases such as clinical arrhythmia and hitting upon treatment strategies based on this.

\section{Methods}

\section{1) Mice}

All Mice were handled in accordance with the guidelines of the Institutional Animal Care and Use Committee of Laboratory Animal Sciences, Jinan University. Mice were maintained on a C129/C57BL6/J mixed background. Both male and female mice were randomized in different experiment groups in this study. The morning of vaginal plug detection was designated as E0.5 [13]. Caesarean section was performed on pregnant mice receiving tamoxifen to obtain perinatal pups. Tamoxifen (Sigma, T5648-5G) was dissolved in corn oil $(20 \mathrm{mg} / \mathrm{ml})$ and introduced by gavage at indicated time $(0.1-0.16 \mathrm{mg}$ per gram mouse body weight). Nppa-GFP mouse line was generated by homologous recombination using Red/ET recombineering as previously described [14]. For Nppa-GFP mouse lines and cDNAs-encoding DTRGFP fusion protein were inserted into frame with the translational start codon of the Nppa gene [15]. For Nppa-CreER allele, a cDNA encoding Cre recombinase fused with a mutant form of the estrogen receptor hormone-binding domain (CreERT2) [16] was inserted into the translational stop codon of Nppa gene (before 3' UTR), with a self-cleaving 2 A peptide sequence linking Nppa and CreER. The process of confirmation of the correct targeted clones and normal karyotype are as previously described standard protocols [17]. For germline transmission, C57B/6 lines were crossed with the obtained chimeric mouse lines. Knock-in mice used here were generated by Shanghai Biomodel Organism Science \& Technology Development Co. Ltd.

\section{2) Genomic PCR}

Genomic DNA was prepared from embryonic yolk sac or mouse tail. Tissues were lysed by incubation with proteinase $\mathrm{K}$ overnight at $55^{\circ} \mathrm{C}$, followed by centrifugation for $8 \mathrm{~min}$ to obtain supernatant with genomic DNA. DNA was precipitated by adding isopropanol and was washed in $70 \%$ ethanol. All embryos and mice were genotyped with specific primers that distinguished the knock-in allele from the wild-type allele [18].

\section{3) Stereo Microscopy}

Caesarean sectioning and cross-fostering of the mouse and heart sampling for stereo microscopy was prepared according to previous protocols [19]. Briefly, embryonic hearts from timed pregnancies and neonatal hearts of P0, P3, P5, P7, P28 and 8W were dissected in PBS and fixed in 4\% paraformaldehyde (PFA) for 15 min to 1 hour depending on the size of hearts at $4^{\circ} \mathrm{C}$. After wash with PBS for three times, whole mount bright-field and fluorescence view images were cap- 
tured under Leica Stereomicroscope (M205 FA/DFC 7000T).

\section{4) Immunostaining on cryosections}

Immunostaining was performed according to previously described protocols [20]. Briefly, after stereomicroscopy processing, obtained fluorescence hearts were transferred to $30 \%$ sucrose for overnight cryopreservation at $4^{\circ} \mathrm{C}$ and embedded in OCT (Sakura). Tissues were collected at $10 \mu \mathrm{m}$ thick on positively charged adhesion microscope slides (CITOTEST) on cryotome (Thermo HM525 NX). Sections were cryo-sectioned to reveal the coronary plane of atrial. PBS supplemented with $0.2 \%$ triton X-100 and 5\% normal donkey serum (Jackson ImmunoResearch) were used for blocking at room temperature for 1 hour, followed by first antibody incubation at $4^{\circ} \mathrm{C}$ overnight. For endogenous antigens detection, commercial antibodies were used: GFP (Abcam, Invitrogen, Nacalai tesque Inc., 1:500), tdTomato (Rockland, 1:5000), TNNI3 (Abcam, 1:200). Fluorescence signals were amplified with Alexa fluorescence antibodies. Immunostaining images were photographed by Olympus fluorescence microscope (FV3000) and Leica stereomicroscope (M205 FA/DFC 7000T).

\section{Acknowledgements}

We thank all colleagues and their support from Key Laboratory of Regenerative Medicine of Ministry of Education. College of Life Science and Technology, Jinan University, Guangzhou, China.

\section{Conflicts of Interest}

The author declares no conflicts of interest regarding the publication of this paper.

\section{References}

[1] Moorman, A.F.M. and Christoffels, V.M. (2003) Cardiac Chamber Formation: Development, Genes, and Evolution. Physiological Reviews, 83, 1223-1267. https://doi.org/10.1152/physrev.00006.2003

[2] Meilhac, S.M., et al. (2004) The Clonal Origin of Myocardial Cells in Different Regions of the Embryonic Mouse Heart. Developmental Cell, 6, 685-698. https://doi.org/10.1016/S1534-5807(04)00133-9

[3] Abu-Issa, R. and Kirby, M.L. (2008) Patterning of the Heart Field in the Chick. Developmental Biology, 319, 223-233. https://doi.org/10.1016/j.ydbio.2008.04.014

[4] Liu, Q.Z., et al. (2016) Genetic Lineage Tracing Identifies in Situ Kit-Expressing Cardiomyocytes. Cell Research, 26, 119-130. https://doi.org/10.1038/cr.2015.143

[5] Lin, Q., et al. (1997) Control of Mouse Cardiac Morphogenesis and Myogenesis by Transcription Factor MEF2C. Science, 276, 1404-1407. https://doi.org/10.1126/science.276.5317.1404

[6] Yelon, D., Horne, S.A. and Stainier, D.Y.R. (1999) Restricted Expression of Cardiac Myosin Genes Reveals Regulated Aspects of Heart Tube Assembly in Zebrafish. Developmental Biology, 214, 23-37. https://doi.org/10.1006/dbio.1999.9406

[7] Gupta, V. and Poss, K.D. (2012) Clonally Dominant Cardiomyocytes Direct Heart Morphogenesis. Nature, 484, 479-484. https://doi.org/10.1038/nature11045 
[8] Tian, X.Y., et al. (2017) Identification of a Hybrid Myocardial Zone in the Mammalian Heart after Birth. Nature Communications, 8, Article No. 87. https://doi.org/10.1038/s41467-017-00118-1

[9] Habets, E.M.H., et al. (2002) Cooperative Action of Tbx2 and Nkx2.5 Inhibits ANF Expression in the Atrioventricular Canal: Implications for Cardiac Chamber Formation. Genes \& Development, 16, 1234-1246. https://doi.org/10.1101/gad.222902

[10] Naksuk, N., et al. (2016) Left Atrial Appendage: Embryology, Anatomy, Physiology, Arrhythmia and Therapeutic Intervention. JACC: Clinical Electrophysiology, 2, 403412. https://doi.org/10.1016/j.jacep.2016.06.006

[11] Słodowska, K., et al. (2021) Morphology of the Left Atrial Appendage: Introduction of a New Simplified Shape-Based Classification System. Heart, Lung and Circulation, 30, 1014-1022. https://doi.org/10.1016/j.hlc.2020.12.006

[12] Wijesurendra, R.S. and Casadei, B. (2019) Mechanisms of Atrial Fibrillation. Heart, 105, Article No. 1860. https://doi.org/10.1136/heartjnl-2018-314267

[13] Tian, X., et al. (2020) Generation of a Self-Cleaved Inducible Cre Recombinase for Efficient Temporal Genetic Manipulation. The EMBO Journal, 39, e102675.

https://doi.org/10.15252/embj.2019102675

[14] Liu, P.T., Jenkins, N.A. and Copeland, N.G. (2003) A Highly Efficient Recombineering-Based Method for Generating Conditional Knockout Mutations. Genome Research, 13, 476-484. https://doi.org/10.1101/gr.749203

[15] Jung, S., et al. (2002) In Vivo Depletion of CD11c ${ }^{+}$Dendritic Cells Abrogates Priming of CD8 ${ }^{+} \mathrm{T}$ Cells by Exogenous Cell-Associated Antigens. Immunity, 17, 211-220. https://doi.org/10.1016/S1074-7613(02)00365-5

[16] Indra, A.K., et al. (1999) Temporally-Controlled Site-Specific Mutagenesis in the Basal Layer of the Epidermis: Comparison of the Recombinase Activity of the Tamoxifen-Inducible Cre-ERT and Cre-ERT2 Recombinases. Nucleic Acids Research, 27, 4324-4327. https://doi.org/10.1093/nar/27.22.4324

[17] Liu, Q., et al. (2015) c-kit ${ }^{+}$Cells Adopt Vascular Endothelial but Not Epithelial Cell Fates during Lung Maintenance and Repair. Nature Medicine, 21, 866-868. https://doi.org/10.1038/nm.3888

[18] Zhang, H., et al. (2016) Genetic Lineage Tracing Identifies Endocardial Origin of Liver Vasculature. Nature Genetics, 48, 537-543. https://doi.org/10.1038/ng.3536

[19] Cai, Z.P., et al. (2018) Caesarean Sectioning and Cross-Fostering of the Mouse. Bio-Protocol, 8, e3085. https://doi.org/10.21769/BioProtoc.3085

[20] Tian, X.Y., et al. (2014) De Novo Formation of a Distinct Coronary Vascular Population in Neonatal Heart. Science, 345, 90-94. https://doi.org/10.1126/science.1251487 